\title{
Stochastic methods for light propagation and recurrent scattering in saturated and nonsaturated atomic ensembles
}

\author{
Mark D. Lee, Stewart D. Jenkins, and Janne Ruostekoski \\ Mathematical Sciences, University of Southampton, Southampton SO17 1BJ, United Kingdom
}

(Dated: March 23, 2016)

\begin{abstract}
We derive equations for the strongly coupled system of light and dense atomic ensembles. The formalism includes an arbitrary internal level structure for the atoms and is not restricted to weak excitation of atoms by light. In the low light intensity limit for atoms with a single electronic ground state, the full quantum field-theoretical representation of the model can be solved exactly by means of classical stochastic electrodynamics simulations for stationary atoms that represent cold atomic ensembles. Simulations for the optical response of atoms in a quantum degenerate regime require one to synthesize a stochastic ensemble of atomic positions that generate the corresponding quantum statistical position correlations between the atoms. In the case of multiple ground levels or at light intensities where saturation becomes important, the classical simulations require approximations that neglect quantum fluctuations between the levels. We show how the model is extended to incorporate corrections due to quantum fluctuations that result from virtual scattering processes. In the low light intensity limit we illustrate the simulations in a system of atoms in a Mott-insulator state in a 2D optical lattice, where recurrent scattering of light induces strong interatomic correlations. These correlations result in collective many-atom sub- and super-radiant states and a strong dependence of the response on the spatial confinement within the lattice sites.
\end{abstract}

PACS numbers: $42.50 . \mathrm{Nn}, 42.50 . \mathrm{Ct}, 42.25 . \mathrm{Bs}, 32.70 . \mathrm{Jz}$

\section{INTRODUCTION}

Interactions mediated by electromagnetic fields can induce correlations between resonant emitters in systems that would not otherwise show any correlation effects. This behavior generally occurs when the resonant dipoledipole interactions between the emitters are sufficiently strong - when the spacing between emitters in homogeneously broadened samples is less than a wavelength [1].

Such cooperative phenomena [1-25] cannot be described by a model of dipoles which scatter independently. They are intimately linked to the presence of recurrent (or dependent) scattering; when a photon scatters more than once off the same emitter. The resulting interactions produce collective excitation eigenmodes, each with a distinct resonance frequency and decay rate. Some of these modes are superradiant, in which case the emission rates compared with those of a single isolated atom are enhanced by collective interactions. In other modes, the radiative emission is subradiant with the collective emission rates that are weaker than that of an isolated atom. In a strongly-coupled response of large ensembles of resonant emitters the ratio between the most sub- and super-radiant decay rates can exceed several orders of magnitude $[9,16]$ if the average separation between the emitters is sufficiently small. As well as the magnitude of the atom density, the response can be sensitive to details such as atom statistics $[6,11,26]$, the degree of inhomogeneous broadening [21,27], or spatial disorder that can lead to light localization [28]. Lightinduced correlations between the atoms due to resonant dipole-dipole interactions can be entirely classical and may result in a failure [21] of standard textbook theories $[29,30]$ of the electrodynamics of a polarizable medium that are based on effective continuous medium models.

High resolution spectroscopic experiments can now be performed with optically dense atomic ensembles at increasing atom densities [22, 31-36]. It has been predicted that correlation phenomena due to light-mediated interactions can emerge in cold atomic ensembles already at surprisingly low atom densities [1]. In particular, in cold alkali-metal atomic gases with densities of over $10^{14} \mathrm{~cm}^{-3}$ the observation of correlated scattering should be achievable [22, 31]. Strong dipole-dipole interactions in cold ensembles of highly-excited Rydberg atoms [37-41] also provide a possible platform to explore cooperative coupling between atoms. Strong dipole-dipole interactions may need to be considered to an increasing extent in quantum technologies. For example, collective phenomena, such as superradiant emission, can be important in implementations of quantum memories and quantum interfaces between light and cold atom systems [42]. The advent of metamaterials, arrays of artificially fabricated magnetodielectric resonators whose electromagnetic response and positions are controlled by design, provide a way to exploit cooperative interactions, for example, to focus light or image a system beyond the diffraction limit [43], to produce narrow transmission resonances [44-46], or to create a broad area coherent light source driven by an electron beam [47].

Previous studies of light-atom interactions have provided a full field-theoretical formulation $[7,8]$ where the atoms and light were treated in terms of quantum fields throughout the analysis. It was shown, specifically in the limit of low light intensity, that the strong light-matter coupling leads to a hierarchy of equations of motion for the correlation functions of atomic density and polariza- 
tion. In the resulting hierarchy, atomic polarization density is coupled to a two-atom correlation function which is then coupled to a three-atom correlation function, etc. General solutions to such a hierarchy of equations even in the case of small atom numbers are challenging, although perturbative solutions may be derived, e.g., in inhomogeneously broadened samples [21], or in the limit of low atom density $\rho$ when $\rho / k^{3} \ll 1[6,11,26]$, where $k$ denotes the resonant wave number of light. An alternative approach, based on a stochastic Monte-Carlo simulation technique, was presented in Ref. [10] by considering a simplified 1D scalar electrodynamics of light-atom interactions. The dynamics of the hierarchy for the equations of motion in the limit of low light intensity was reproduced to all orders by solving a system of classical electrodynamics equations for point dipoles at fixed positions - these spatial positions are then stochastically sampled from appropriate spatial distributions for each realization (for a classical gas the atomic positions are independently distributed and for quantum degenerate atoms they are sampled from the joint many-body probability distribution). Such a coupled dipole model between pointlike atoms was explicitly shown to provide an exact solution for the low light intensity scalar theory with stationary atoms at arbitrary atom densities by means of a classical electrodynamics simulation where all the recurrent scattering processes are fully accounted for. Analogous techniques to simulate classical electrodynamics of systems comprising discrete emitters have been applied to a variety of systems, for example, to scatterers at fixed positions $[16,45]$, and to scatterers whose positions are stochastically distributed $[1,14,15,17,19,21,48]$. These simulations considered the low light intensity limit and involved either emitters with an isotropic polarizability or emitters whose dipole orientations were all fixed in the same direction (e.g., two-level atoms) or were averaged over the spatial directions. Situations typically encountered in experiments on cold atomic ensembles, however, involve atoms with an internal hyperfine level structure and are not limited only to low light intensities.

In this paper we derive general stochastic simulations techniques for the light-atom interactions in cold atomic ensembles based on a quantum field-theoretical analysis for the coupled theory of atoms and light. We incorporate a multiple level structure of the atoms, full vector properties of the electromagnetic fields, and saturation of the atoms due to illuminating light. We show that the Monte-Carlo simulation technique, and the corresponding equations of motion for the atoms in each stochastic realization, can be derived by a methodologically simple procedure that is based on taking expectation values of the atoms when they are conditioned on a given set of fixed spatial positions. This allows us to show explicitly when the entire optical response of the atomic ensemble can be represented exactly by a relatively simple set of coupled equations for the atomic dipoles and when more complex theories are required in order to deal with classical and quantum-mechanical light-induced correla- tions between the atoms. We specifically show that in the low light intensity limit and for atoms with only a single electronic ground state, the full optical response can be represented exactly (within the statistical accuracy) in terms of classical electrodynamics equations of motion for coupled point dipoles driven by an incident field, when the positions of the dipoles are stochastically sampled according to the statistical position correlations of the atoms and ensemble averaged over many such realizations. In the presence of a Zeeman level structure where multiple electronic ground states can couple to the light, we show that the derivation of similar classical electrodynamics equations of motion requires an approximate treatment of fluctuations due to repeated virtual photon exchanges between atoms occupying different ground levels. We introduce a classical approximation where the recurrent (or dependent) scattering between the atoms is included, but nonclassical higher order correlations between internal levels that involve fluctuations between ground-state coherences of different levels and the polarization are factorized. The classical approximation correctly reproduces all the atomic ground-state population expectation values that, in the limit of low light intensity, remain constant (unchanged from their initial values) even in the exact quantum solution. We show how one can go beyond this level of approximation by progressively incorporating higher order quantum fluctuation effects.

Our derivation for the stochastic techniques for the electrodynamics simulations is not only restricted to the low light intensity limit. Once saturation becomes important and the population of the electronically excited levels can no longer be neglected, we show that we can adapt the stochastic simulations to provide a tractable coupled-dipole approach that scales favorably with the atom number and where the internal level dynamics is treated semiclassically. This is obtained by using factorization approximations for certain correlation functions, in a manner analogous to the factorization approximation introduced in the low light intensity case of multiple ground levels.

In order to illustrate briefly the stochastic electrodynamics simulations in practise, we also consider a specific numerical example. In the low light intensity limit, we calculate the optical response and collective excitation eigenmodes of an atomic gas confined in a $2 \mathrm{D}$ optical lattice by extending the model introduced in Ref. [17], where the control of atomic positions was used to engineer highly localized subwavelength-scale optical excitations in the atomic system. We show that the system supports collective excitation eigenmodes that exhibit a broad range of super- and sub-radiant decay rates, and we examine how this distribution depends on the lattice spacing and confinement strength. Classical electrodynamics simulations that treat multilevel ${ }^{87} \mathrm{Rb}$ atoms for fluorescence and coherent light transmission are reported elsewhere [22, 49, 50].

In Sec. II we present the formalism necessary to de- 
scribe the collective scattering problem for atoms in free space, and derive general expressions for the optical response. We then introduce the Monte-Carlo sampling method, firstly for low light intensity in Sec. III, before including saturation in Sec. IV. Finally, we provide the numerical example in Sec. V.

\section{GENERAL THEORY}

In this section we derive equations for the collective optical response of an ensemble of atoms. We start with a quantum field-theoretical formalism introduced in Refs. [7, 8, 51], and extend the formalism beyond the low light-intensity limit and by systematically including multiple internal level structure.

\section{A. Hamiltonian}

We begin with a nonrelativistic Hamiltonian formalism of electrodynamics in the dipole approximation for atoms, expressing the Hamiltonian in the length gauge obtained in the Power-Zienau-Woolley transformation $[52-55]$. The atoms and light are treated in terms of quantum fields throughout the analysis. In second quantization formalism for the electronic ground and excited states of the atoms we account for the sublevel structure, so that the corresponding field operators read $\hat{\psi}_{g \nu}(\mathbf{r})$ and $\hat{\psi}_{e \eta}(\mathbf{r})$, respectively. Here the second subscript refers to the sublevel index, such that the complete set of atomic basis states is formed by $|g, \nu\rangle$ and $|e, \eta\rangle$.

We write the Hamiltonian density as

$$
\mathcal{H}=\mathcal{H}_{g}+\mathcal{H}_{e}+\mathcal{H}_{F}+\mathcal{H}_{D}+\mathcal{H}_{P} .
$$

The atomic internal level energy is expressed by the first two terms, for the example of the linear Zeeman shift caused by a magnetic field of strength $B$ these are given by

$$
\begin{aligned}
& \mathcal{H}_{g}=\hat{\psi}_{g \nu}^{\dagger}(\mathbf{r})\left(\mu_{B} B g_{l}^{(g)} \nu\right) \hat{\psi}_{g \nu}(\mathbf{r}), \\
& \mathcal{H}_{e}=\hat{\psi}_{e \eta}^{\dagger}(\mathbf{r})\left(\mu_{B} B g_{l}^{(e)} \eta+\hbar \omega_{0}\right) \hat{\psi}_{e \eta}(\mathbf{r}),
\end{aligned}
$$

where $g_{l}^{(g)}$ and $g_{l}^{(e)}$ are the Landé g-factors [56] for levels $g$ and $e$, and $\omega_{0}$ is the resonance frequency of the $|g\rangle \leftrightarrow|e\rangle$ transition in the absence of any magnetic field.

For the electromagnetic field we introduce the mode frequency and the photon annihilation operator as $\omega_{q}$ and $\hat{a}_{q}$, respectively. The mode index $q$ incorporates both the wave vector $\mathbf{q}$ and the transverse polarization $\hat{\mathbf{e}}_{q}$. In the length gauge it is beneficial to represent the light amplitude by the electric displacement $\hat{\mathbf{D}}(\mathbf{r})$ which is the basic dynamical variable for light [55]. We write it as a sum of the positive and negative frequency components $\hat{\mathbf{D}}=\hat{\mathbf{D}}^{+}+\hat{\mathbf{D}}^{-}$, with $\hat{\mathbf{D}}^{+}=\left[\hat{\mathbf{D}}^{-}\right]^{\dagger}$, and with

$$
\hat{\mathbf{D}}^{+}(\mathbf{r})=\sum_{q} \zeta_{q} \hat{\mathbf{e}}_{q} \hat{a}_{q} e^{i \mathbf{q} \cdot \mathbf{r}}, \quad \zeta_{q}=\sqrt{\frac{\hbar \epsilon_{0} \omega_{q}}{2 V}},
$$

where $V$ denotes the quantization volume. The Hamiltonian for the free electromagnetic field energy then reads

$$
H_{F}=\int d^{3} r \mathcal{H}_{F}=\sum_{q} \hbar \omega_{q} \hat{a}_{q}^{\dagger} \hat{a}_{q}
$$

The atomic polarization $\hat{\mathbf{P}}(\mathbf{r})$ interacts with the electric displacement and we have

$$
\mathcal{H}_{D}=-\frac{1}{\epsilon_{0}} \hat{\mathbf{P}}(\mathbf{r}) \cdot \hat{\mathbf{D}}(\mathbf{r})
$$

We also have in the Hamiltonian the polarization selfenergy term:

$$
\mathcal{H}_{\mathrm{P}}=\frac{1}{2 \epsilon_{0}} \hat{\mathbf{P}}(\mathbf{r}) \cdot \hat{\mathbf{P}}(\mathbf{r}) .
$$

For nonoverlapping atomic point dipoles such a term vanishes.

In terms of our second quantized field operators, the positive frequency component of the polarization can be written in terms of contributions from differing sublevel transitions as

$$
\begin{aligned}
\hat{\mathbf{P}}^{+}(\mathbf{r}) & =\sum_{\nu, \eta} \mathbf{d}_{g \nu e \eta} \hat{\psi}_{g \nu}^{\dagger}(\mathbf{r}) \hat{\psi}_{e \eta}(\mathbf{r}) \equiv \sum_{\nu, \eta} \hat{\mathbf{P}}_{\nu \eta}^{+}(\mathbf{r}), \\
\hat{\mathbf{P}}_{\nu \eta}^{+}(\mathbf{r}) & \equiv \mathbf{d}_{g \nu e \eta} \hat{\psi}_{g \nu}^{\dagger}(\mathbf{r}) \hat{\psi}_{e \eta}(\mathbf{r}),
\end{aligned}
$$

where $\mathbf{d}_{g \nu e \eta}$ represents the dipole matrix element for the transition $|e, \eta\rangle \rightarrow|g, \nu\rangle$

$$
\mathbf{d}_{g \nu e \eta} \equiv \mathcal{D} \sum_{\sigma} \hat{\mathbf{e}}_{\sigma}\langle e \eta ; 1 g \mid 1 \sigma ; g \nu\rangle \equiv \mathcal{D} \sum_{\sigma} \hat{\mathbf{e}}_{\sigma} \mathcal{C}_{\nu, \eta}^{(\sigma)}
$$

Here the sum is over the unit circular polarization vectors $\sigma= \pm 1,0$, and $\mathcal{C}_{\nu, \eta}^{(\sigma)}$ denote the Clebsch-Gordan coefficients of the corresponding optical transitions. The reduced dipole matrix element is represented by $\mathcal{D}$ (here chosen to be real) and $\mathbf{d}_{e \eta g \nu}=\mathbf{d}_{g \nu e \eta}^{*}$. The light fields with the polarizations $\hat{\mathbf{e}}_{ \pm}$and $\hat{\mathbf{e}}_{0}$ drive the transitions $|g, \nu\rangle \rightarrow|e, \nu \pm 1\rangle$ and $|g, \nu\rangle \rightarrow|e, \nu\rangle$, respectively, in such a way that only the terms $\sigma=\eta-\nu$ in Eq. (10) are nonvanishing.

\section{B. Light and matter fields}

We assume that the atoms are illuminated by a nearmonochromatic incident laser at the frequency $\Omega$. In the following we assume that all the relevant quantities are expressed as slowly varying amplitudes by explicitly factoring out the dominant laser frequency oscillations by writing $\hat{\psi}_{e \eta} \rightarrow e^{-i \Omega t} \hat{\psi}_{e \eta}, \hat{\mathbf{D}}^{+} \rightarrow e^{-i \Omega t} \hat{\mathbf{D}}^{+}$, etc.

\section{Scattered light}

The light field may be obtained by integrating the equations for motion for the photon operator $\hat{a}_{q}$, derived 
from the full Hamiltonian. The electric displacement can be expressed as a sum $\hat{\mathbf{D}}^{+}(\mathbf{r})=\hat{\mathbf{D}}_{F}^{+}(\mathbf{r})+\hat{\mathbf{D}}_{S}^{+}(\mathbf{r})$ of the free field part $\hat{\mathbf{D}}_{F}^{+}(\mathbf{r})$ that would exist in the absence of the matter, and the scattered field $\hat{\mathbf{D}}_{S}^{+}(\mathbf{r})$. From

$$
\hat{\mathbf{D}}(\mathbf{r})=\epsilon_{0} \hat{\mathbf{E}}(\mathbf{r})+\hat{\mathbf{P}}(\mathbf{r})
$$

we can write the electric field $\epsilon_{0} \hat{\mathbf{E}}^{+}(\mathbf{r})=\hat{\mathbf{D}}_{F}^{+}(\mathbf{r})+\hat{\mathbf{D}}_{S}^{+}(\mathbf{r})-$ $\hat{\mathbf{P}}^{+}(\mathbf{r})$ as the integral expression [7] $(k=\Omega / c)$

$$
\begin{aligned}
\epsilon_{0} \hat{\mathbf{E}}^{+}(\mathbf{r}) & =\hat{\mathbf{D}}_{F}^{+}(\mathbf{r})+\int d^{3} r^{\prime} \mathrm{G}\left(\mathbf{r}-\mathbf{r}^{\prime}\right) \hat{\mathbf{P}}^{+}\left(\mathbf{r}^{\prime}\right), \\
\mathbf{G}_{i j}(\mathbf{r}) & =\left[\frac{\partial}{\partial r_{i}} \frac{\partial}{\partial r_{j}}-\delta_{i j} \nabla^{2}\right] \frac{e^{i k r}}{4 \pi r}-\delta_{i j} \delta(\mathbf{r}) .
\end{aligned}
$$

In Eq. (12) the monochromatic dipole radiation kernel $\mathrm{G}(\mathbf{r})$ [29] provides the radiated field at $\mathbf{r}$ from a dipole with the amplitude $\hat{\mathbf{d}}$ residing at the origin:

$$
\begin{aligned}
& \mathrm{G}(\mathbf{r}) \hat{\mathbf{d}}=\frac{k^{3}}{4 \pi}\left\{(\hat{\mathbf{n}} \times \hat{\mathbf{d}}) \times \hat{\mathbf{n}} \frac{e^{i k r}}{k r}\right. \\
& \left.\quad+[3 \hat{\mathbf{n}}(\hat{\mathbf{n}} \cdot \hat{\mathbf{d}})-\hat{\mathbf{d}}]\left[\frac{1}{(k r)^{3}}-\frac{i}{(k r)^{2}}\right] e^{i k r}\right\}-\frac{\hat{\mathbf{d}} \delta(\mathbf{r})}{3}
\end{aligned}
$$

where $\hat{\mathbf{n}}=\mathbf{r} / r$. When integrating over a volume including the origin, the term in Eq. (14) proportional to $1 / r^{3}$ does not absolutely converge. Following Ref. [7] we interpret Eq. (14) in such a way that the integral over an infinitesimal volume enclosing the origin of the term inside the curly brackets vanishes.

\section{Evolution of atomic fields}

We may also derive the equations of motion for the excited and ground state atomic fields

$$
\begin{aligned}
& \dot{\hat{\psi}}_{e \eta}(\mathbf{r})=i \Delta_{e \eta} \hat{\psi}_{e \eta}(\mathbf{r})+\frac{i}{\hbar} \mathbf{d}_{e \eta g \nu} \cdot \hat{\mathbf{E}}^{+}(\mathbf{r}) \hat{\psi}_{g \nu}(\mathbf{r}), \\
& \dot{\hat{\psi}}_{g \nu}(\mathbf{r})=i \Delta_{g \nu} \hat{\psi}_{g \nu}(\mathbf{r})+\frac{i}{\hbar} \hat{\mathbf{E}}^{-}(\mathbf{r}) \cdot \mathbf{d}_{g \nu e \eta} \hat{\psi}_{e \eta}(\mathbf{r}) .
\end{aligned}
$$

Here in Eq. (15) we implicitly sum over all $\nu$ and in Eq. (16) over all $\eta$. Similarly, in the remainder of the paper we take repeated indices in expressions to indicate an implicit summation. The atom-light detuning is denoted by $\Delta_{e \eta}=\Omega-\left(\omega_{0}+\mu_{B} B g_{l}^{(e)} \eta / \hbar\right)$ and $\Delta_{g \nu}=-\mu_{B} B g_{l}^{(g)} \nu / \hbar$. For reasons that become obvious later on, we would like to place all the atomic field operators in the normal order. In order to evaluate the free field operator expectation values, we will place $\hat{\mathbf{D}}_{F}^{+}(\mathbf{r})$ farthest to right and $\hat{\mathbf{D}}_{F}^{-}(\mathbf{r})$ farthest to the left. Since the initial free field is assumed to be in a coherent state, the expectation values then produce multiplicative classical coherent fields [57]. Using Eq. (12), for (15) we obtain

$$
\begin{array}{r}
\dot{\hat{\psi}}_{e \eta}(\mathbf{r})=i \Delta_{e \eta} \hat{\psi}_{e \eta}(\mathbf{r})+\frac{i}{\hbar \epsilon_{0}} \mathbf{d}_{e \eta g \nu} \cdot \hat{\mathbf{D}}_{F}^{+}(\mathbf{r}) \hat{\psi}_{g \nu}(\mathbf{r}) \\
+\frac{i}{\hbar \epsilon_{0}} \mathbf{d}_{e \eta g \nu} \cdot \int d^{3} r^{\prime} \mathrm{G}\left(\mathbf{r}-\mathbf{r}^{\prime}\right) \hat{\mathbf{P}}^{+}\left(\mathbf{r}^{\prime}\right) \hat{\psi}_{g \nu}(\mathbf{r}) .
\end{array}
$$

We show in App. A that we can evaluate the commutator

$$
\left[\hat{\mathbf{D}}_{F}^{+}(\mathbf{r}), \hat{\psi}_{g \nu}\left(\mathbf{r}^{\prime}\right)\right]=\left[\mathrm{G}\left(\mathbf{r}-\mathbf{r}^{\prime}\right)+\delta\left(\mathbf{r}-\mathbf{r}^{\prime}\right)\right] \mathbf{d}_{g \nu e \zeta} \hat{\psi}_{e \zeta}\left(\mathbf{r}^{\prime}\right) .
$$

Taking the limit $\mathbf{r}^{\prime} \rightarrow \mathbf{r}$ in the field-theory version of the Born and Markov approximations [7], and substituting into (17) yields

$$
\begin{gathered}
\dot{\hat{\psi}}_{e \eta}(\mathbf{r})=\left(i \Delta_{e \eta}-\gamma\right) \hat{\psi}_{e \eta}(\mathbf{r})+\frac{i}{\hbar \epsilon_{0}} \hat{\psi}_{g \nu}(\mathbf{r}) \mathbf{d}_{e \eta g \nu} \cdot \hat{\mathbf{D}}_{F}^{+}(\mathbf{r}) \\
+\frac{i}{\hbar \epsilon_{0}} \mathbf{d}_{e \eta g \nu} \cdot \int d^{3} r^{\prime} G^{\prime}\left(\mathbf{r}-\mathbf{r}^{\prime}\right) \hat{\mathbf{P}}^{+}\left(\mathbf{r}^{\prime}\right) \hat{\psi}_{g \nu}(\mathbf{r}) .
\end{gathered}
$$

The spontaneous linewidth $\gamma$ results from the imaginary part of the commutator limit and is given by

$$
\gamma \equiv \frac{\mathcal{D}^{2} k^{3}}{6 \pi \hbar \epsilon_{0}}
$$

On the other hand, the divergent real part of the commutator limit contributes to the Lamb shift and is assumed to be included in the detuning $\Delta$. We have introduced the propagator

$$
\mathrm{G}_{i j}^{\prime}(\mathbf{r}) \equiv \mathrm{G}_{i j}(\mathbf{r})+\frac{\delta_{i j} \delta(\mathbf{r})}{3},
$$

explicitly canceling the contact term in $G(\mathbf{r})$, such that the integral of $\mathrm{G}^{\prime}(\mathbf{r})$ over an infinitesimal volume about the origin vanishes. Since the hard-core of real interatomic potentials prevents the overlap of cold atoms, the contact term is inconsequential for real applications. In fact, even for ideal point dipoles it can be shown in the low light intensity limit that the light matter dynamics is independent of the contact term [8].

In the equation of motion for $\hat{\psi}_{g \nu}(\mathbf{r})$ the free field is already in the correct order and we obtain

$$
\begin{gathered}
\dot{\hat{\psi}}_{g \nu}(\mathbf{r})=i \Delta_{g \nu} \hat{\psi}_{g \nu}(\mathbf{r})+\frac{i}{\hbar \epsilon_{0}} \hat{\mathbf{D}}_{F}^{-}(\mathbf{r}) \cdot \mathbf{d}_{g \nu e \eta} \hat{\psi}_{e \eta}(\mathbf{r}) \\
+\frac{i}{\hbar \epsilon_{0}} \mathbf{d}_{g \nu e \eta} \cdot \int d^{3} r^{\prime} \mathrm{G}^{\prime *}\left(\mathbf{r}-\mathbf{r}^{\prime}\right) \hat{\mathbf{P}}^{-}\left(\mathbf{r}^{\prime}\right) \hat{\psi}_{e \eta}(\mathbf{r}) .
\end{gathered}
$$

When we derive the evolution equations for the atomic polarization and the level populations that incorporate the internal level structure of the atoms, it is useful to introduce the following tensor

$$
\mathrm{P}_{\mu \tau}^{\nu \eta} \equiv \frac{\mathbf{d}_{g \nu e \eta} \mathbf{d}_{e \mu g \tau}}{\mathcal{D}^{2}}=\sum_{\sigma, \varsigma} \hat{\mathbf{e}}_{\sigma} \hat{\mathbf{e}}_{\varsigma}^{*} \mathcal{C}_{\nu, \eta}^{(\sigma)} \mathcal{C}_{\tau, \mu}^{(\varsigma)} .
$$

The components of $\mathrm{P}_{\mu \tau}^{\nu \eta}$ depend on the vector properties of the atomic level structure weighted by the ClebschGordan coefficients (here taken to be real). 
Combining Eqs. (19) and (22), and rewriting in normal order, the optical response of the system can be encoded in the equations of motion for the polarization operator component $\hat{\mathbf{P}}_{\nu \eta}^{+}(\mathbf{r})$, and ground and excited state coherence operators $\hat{\psi}_{g \nu}^{\dagger} \hat{\psi}_{g \eta}, \hat{\psi}_{e \nu}^{\dagger} \hat{\psi}_{e \eta}$

$$
\begin{aligned}
& \frac{d}{d t} \hat{\mathbf{P}}_{\nu \eta}^{+}=\left(i \bar{\Delta}_{g \nu e \eta}-\gamma\right) \hat{\mathbf{P}}_{\nu \eta}^{+}+i \xi \hat{\psi}_{g \nu}^{\dagger} \hat{\psi}_{g \tau} \mathrm{P}_{\eta \tau}^{\nu \eta} \hat{\mathbf{D}}_{F}^{+} \\
& +i \xi \int d^{3} r^{\prime} \mathrm{P}_{\eta \tau}^{\nu \eta} \mathrm{G}^{\prime}\left(\mathbf{r}-\mathbf{r}^{\prime}\right) \hat{\psi}_{g \nu}^{\dagger} \hat{\mathbf{P}}^{+}\left(\mathbf{r}^{\prime}\right) \hat{\psi}_{g \tau} \\
& -i \xi \int d^{3} r^{\prime} \mathbf{P}_{\tau \nu}^{\nu \eta} \mathrm{G}^{\prime}\left(\mathbf{r}-\mathbf{r}^{\prime}\right) \hat{\psi}_{e \tau}^{\dagger} \hat{\mathbf{P}}^{+}\left(\mathbf{r}^{\prime}\right) \hat{\psi}_{e \eta} \\
& -i \xi \hat{\psi}_{e \tau}^{\dagger} \hat{\psi}_{e \eta} \mathrm{P}_{\tau \nu}^{\nu \eta} \hat{\mathbf{D}}_{F}^{+} \\
& \frac{d}{d t} \hat{\psi}_{g \nu}^{\dagger} \hat{\psi}_{g \eta}=i \bar{\Delta}_{g \nu g \eta} \hat{\psi}_{g \nu}^{\dagger} \hat{\psi}_{g \eta} \\
& +2 \gamma \mathcal{C}_{\eta,(\eta+\sigma)}^{(\sigma)} \mathcal{C}_{\nu,(\nu+\sigma)}^{(\sigma)} \hat{\psi}_{e(\nu+\sigma)}^{\dagger} \hat{\psi}_{e(\eta+\sigma)} \\
& -i \frac{\xi}{\mathcal{D}^{2}} \hat{\psi}_{e \tau}^{\dagger} \hat{\psi}_{g \eta} \mathbf{d}_{e \tau g \nu} \cdot \hat{\mathbf{D}}_{F}^{+}+i \frac{\xi}{\mathcal{D}^{2}} \hat{\mathbf{D}}_{F}^{-} \cdot \mathbf{d}_{g \eta e \tau} \hat{\psi}_{g \nu}^{\dagger} \hat{\psi}_{e \tau} \\
& -i \frac{\xi}{\mathcal{D}^{2}} \int d^{3} r^{\prime} \mathbf{d}_{e \tau g \nu} \cdot \mathbf{G}^{\prime}\left(\mathbf{r}-\mathbf{r}^{\prime}\right) \hat{\psi}_{e \tau}^{\dagger} \hat{\mathbf{P}}^{+}\left(\mathbf{r}^{\prime}\right) \hat{\psi}_{g \eta} \\
& +i \frac{\xi}{\mathcal{D}^{2}} \int d^{3} r^{\prime} \mathbf{d}_{g \eta e \tau} \cdot \mathbf{G}^{\prime *}\left(\mathbf{r}-\mathbf{r}^{\prime}\right) \hat{\psi}_{g \nu}^{\dagger} \hat{\mathbf{P}}^{-}\left(\mathbf{r}^{\prime}\right) \hat{\psi}_{e \tau}, \\
& \frac{d}{d t} \hat{\psi}_{e \nu}^{\dagger} \hat{\psi}_{e \eta}=\left(i \bar{\Delta}_{e \nu e \eta}-2 \gamma\right) \hat{\psi}_{e \nu}^{\dagger} \hat{\psi}_{e \eta} \\
& +i \frac{\xi}{\mathcal{D}^{2}} \hat{\psi}_{e \nu}^{\dagger} \hat{\psi}_{g \tau} \mathbf{d}_{e \eta g \tau} \cdot \hat{\mathbf{D}}_{F}^{+}-i \frac{\xi}{\mathcal{D}^{2}} \hat{\mathbf{D}}_{F}^{-} \cdot \mathbf{d}_{g \tau e \nu} \hat{\psi}_{g \tau}^{\dagger} \hat{\psi}_{e \eta} \\
& -i \frac{\xi}{\mathcal{D}^{2}} \int d^{3} r^{\prime} \mathbf{d}_{g \tau e \nu} \cdot \mathrm{G}^{\prime *}\left(\mathbf{r}-\mathbf{r}^{\prime}\right) \hat{\psi}_{g \tau}^{\dagger} \hat{\mathbf{P}}^{-}\left(\mathbf{r}^{\prime}\right) \hat{\psi}_{e \eta} \\
& +i \frac{\xi}{\mathcal{D}^{2}} \int d^{3} r^{\prime} \mathbf{d}_{e \eta g \tau} \cdot \mathrm{G}^{\prime}\left(\mathbf{r}-\mathbf{r}^{\prime}\right) \hat{\psi}_{e \nu}^{\dagger} \hat{\mathbf{P}}^{+}\left(\mathbf{r}^{\prime}\right) \hat{\psi}_{g \tau} .
\end{aligned}
$$

Here the repeated indices $\tau, \sigma$ indicate implicit summations over the corresponding sublevels and polarizations, and we define $\bar{\Delta}_{a \nu b \eta}=\Delta_{b \eta}-\Delta_{a \nu}(a, b=g, e)$. In Eqs. (24) we have introduced a notational convention where we show explicitly only the nonlocal position dependence $\mathbf{r}^{\prime}$, and the local coordinate $\mathbf{r}$ of the fields is suppressed. We follow this convention in the rest of the paper whenever the equations would otherwise become notably longer. We have also defined

$$
\xi \equiv \frac{\mathcal{D}^{2}}{\hbar \epsilon_{0}} .
$$

The difficulty in solving the optical response analytically is now evident. Taking expectation values of both sides of Eqs. (24), we see that the one-body correlation functions of interest depend on integrals over two-body correlation functions. In turn, the two-body correlation functions depend on integrals over three-body correlations, and so it continues up to the $N$ th order correlation functions. We give explicit expressions in App. B for the resulting hierarchy of equations of motion for correlation functions in the limit of low light intensity. Outside of this limit, when all correlation functions must be included, the complexity of the coupled equations grows even more rapidly as a function of the number of atoms and internal levels. In practice, even for systems of a few atoms in the low light intensity limit one requires some form of truncation in order to solve the hierarchy of equations. Such approximations to the light-induced correlations may be obtained as a perturbative expansion in the small parameter $\rho / k^{3}$, which is valid at low atom densities $[5,6,11,26]$, or the Doppler broadening of the atoms in inhomogeneously broadened samples [21]. In contrast, the stochastic techniques which we describe in the following sections provide a computationally efficient approach, which in certain situations and limits can be solved exactly at arbitrary atom densities.

In obtaining these equations, we have made the simplification that the atomic motional state is unchanged by the scattering of light, and have ignored the corresponding inelastic processes. In the following sections we describe how stochastic techniques may be used to find the polarization $\left\langle\hat{\mathbf{P}}^{+}(\mathbf{r}, t)\right\rangle$, from which the electric field amplitude $\left\langle\hat{\mathbf{E}}^{+}(\mathbf{r}, t)\right\rangle$ can be calculated using Eq. (12).

Equations (24) are general equations including the effects of saturation and multiple level structure. For twolevel atoms they simplify considerably due to the fixed orientation ê of the atomic dipoles of two-level atoms. As a consequence, the polarization operator of the atoms is proportional to the scalar operator $\hat{P}^{+} \equiv \hat{\mathbf{e}}^{*} \cdot \hat{\mathbf{P}}^{+}$, and components of $\hat{\mathbf{P}}^{+}$orthogonal to $\hat{\mathbf{e}}$ are zero. By making the substitution $\hat{\mathbf{P}}^{+} \rightarrow \hat{\mathbf{e}} \hat{P}^{+}$in Eqs. (24) we obtain the two-level atom expressions

$$
\begin{aligned}
\frac{d}{d t} \hat{P}^{+} & =(i \bar{\Delta}-\gamma) \hat{P}^{+} \\
& +i \xi\left(\hat{\psi}_{g}^{\dagger} \hat{\psi}_{g}-\hat{\psi}_{e}^{\dagger} \hat{\psi}_{e}\right) \hat{\mathbf{e}}^{*} \cdot \hat{\mathbf{D}}_{F}^{+} \\
& +i \xi \int d^{3} r^{\prime} \hat{\mathbf{e}}^{*} \cdot \mathrm{G}^{\prime}\left(\mathbf{r}-\mathbf{r}^{\prime}\right) \hat{\mathbf{e}} \hat{\psi}_{g}^{\dagger} \hat{P}^{+}\left(\mathbf{r}^{\prime}\right) \psi_{g} \\
& -i \xi \int d^{3} r^{\prime} \hat{\mathbf{e}}^{*} \cdot \mathrm{G}^{\prime}\left(\mathbf{r}-\mathbf{r}^{\prime}\right) \hat{\mathbf{e}} \hat{\psi}_{e}^{\dagger} \hat{P}^{+}\left(\mathbf{r}^{\prime}\right) \hat{\psi}_{e} \\
\frac{d}{d t} \hat{\psi}_{g}^{\dagger} \hat{\psi}_{g} & =-\frac{d}{d t} \hat{\psi}_{e}^{\dagger} \hat{\psi}_{e}, \\
& =2 \gamma \hat{\psi}_{e}^{\dagger} \hat{\psi}_{e} \\
& -i \frac{\xi}{\mathcal{D}^{2}} \hat{P}^{-} \hat{\mathbf{e}}^{*} \cdot \hat{\mathbf{D}}_{F}^{+}+i \frac{\xi}{\mathcal{D}^{2}} \hat{\mathbf{D}}_{F}^{-} \cdot \hat{\mathbf{e}} \hat{P}^{+} \\
& -i \frac{\xi}{\mathcal{D}} \int d^{3} r^{\prime} \hat{\mathbf{e}}^{*} \cdot \mathrm{G}^{\prime}\left(\mathbf{r}-\mathbf{r}^{\prime}\right) \hat{\mathbf{e}} \hat{\psi}_{e}^{\dagger} \hat{P}^{+}\left(\mathbf{r}^{\prime}\right) \hat{\psi}_{g} \\
& +i \frac{\xi}{\mathcal{D}} \int d^{3} r^{\prime} \hat{\mathbf{e}}^{\prime} \cdot \mathrm{G}^{*}\left(\mathbf{r}-\mathbf{r}^{\prime}\right) \hat{\mathbf{e}} \hat{\psi}_{g}^{\dagger} \hat{P}^{-}\left(\mathbf{r}^{\prime}\right) \hat{\psi}_{e} \cdot
\end{aligned}
$$

In the limit of low light intensity, the optical response given by Eqs. (24) can be significantly further simplified, particularly so for atoms of isotropic polarizability or two-level atoms. We discuss these simplifications below, before considering the stochastic solution of the resultant equations in Sec. III. We subsequently extend 
the stochastic treatment to the full equations in Sec. IV.

\section{The limit of low light intensity}

In the limit of low light intensity, the atoms occupy the electronic ground states, and the change in the excited state field amplitudes is linearly proportional to the incident light field amplitude, according to Eq. (15). In order to take the weak excitation limit in the first order of the light field amplitude $\hat{\mathbf{D}}_{F}^{ \pm}$we therefore include in the dynamics the leading order contributions given by terms that include at most one of the operators $\hat{\mathbf{D}}_{F}^{ \pm}, \hat{\psi}_{e \eta}$, or $\hat{\psi}_{e \eta}^{\dagger}$. In the first order of light intensity, the excited state population accordingly vanishes. Neglecting higher order terms, we obtain the equation of motion for the polarization operator component $\hat{\mathbf{P}}_{\nu \eta}^{+}(\mathbf{r})$ from Eq. (24a),

$$
\begin{aligned}
\frac{d}{d t} \hat{\mathbf{P}}_{\nu \eta}^{+} & =\left(i \bar{\Delta}_{g \nu e \eta}-\gamma\right) \hat{\mathbf{P}}_{\nu \eta}^{+}+i \xi \hat{\psi}_{g \nu}^{\dagger} \hat{\psi}_{g \tau} \mathrm{P}_{\eta \tau}^{\nu \eta} \hat{\mathbf{D}}_{F}^{+} \\
& +i \xi \int d^{3} r^{\prime} \mathbf{P}_{\eta \tau}^{\nu \eta} \mathrm{G}^{\prime}\left(\mathbf{r}-\mathbf{r}^{\prime}\right) \hat{\psi}_{g \nu}^{\dagger} \hat{\mathbf{P}}^{+}\left(\mathbf{r}^{\prime}\right) \hat{\psi}_{g \tau}
\end{aligned}
$$

On the other hand, any change in the electronic groundstate coherence operators $\hat{\psi}_{g \nu}^{\dagger} \hat{\psi}_{g \eta}$ exhibits merely a phase rotation due to Zeeman splitting. This is because any change in the ground-state amplitude $\hat{\psi}_{g \nu}$ beyond the phase rotation is second order in the incident light field amplitude (via $\mathbf{E}$ and $\hat{\psi}_{\text {en }}$ ) according to Eq. (16).

\section{Isotropic and two-level atoms}

The optical response simplifies further if we consider two special cases of atoms with a single electronic ground level: (1) a gas of atoms whose polarizability is isotropic and (2) a gas of two-level atoms. An isotropic polarizability is obtained when the atoms comprising the gas have a ground state with total electronic and atomic angular momenta $J=0$, such as alkaline-earth-metal or rare-earth metal atoms with zero nuclear spin. By selection rules, the excited state must have angular momentum $J^{\prime}=1$. In the absence of Zeeman splitting between the three different excited sublevels, the atoms exhibit an isotropic polarizability. Two-level systems can be obtained from such atoms by choosing a transition corresponding to an electric dipole along a desired direction. Alternatively, a two-level system can be realized using cycling transitions in alkali-metal atoms. In either scenario, other unwanted transitions could be detuned out of resonance using, for example, static fields to lift the Zeeman degeneracy.

Since only one ground state $|g, \nu=0\rangle$ is present for a gas of atoms with isotropic polarizability, the total polarization of the system is $\hat{\mathbf{P}}^{+}=\sum_{\eta=-1}^{1} \hat{\mathbf{P}}_{0 \eta}^{+}$, and the relevant Clebsch-Gordan coefficients are $\mathcal{C}_{0, \eta}^{(\sigma)}=\delta_{\eta \sigma}$. Consis- tent with Eq. (29), we find the polarization evolves as [7]

$$
\begin{aligned}
\frac{d}{d t} \hat{\mathbf{P}}^{+} & =(i \bar{\Delta}-\gamma) \hat{\mathbf{P}}^{+}+i \xi \hat{\psi}_{g}^{\dagger} \hat{\psi}_{g} \hat{\mathbf{D}}_{F}^{+} \\
& +i \xi \int d^{3} r^{\prime} \mathbf{G}^{\prime}\left(\mathbf{r}-\mathbf{r}^{\prime}\right) \hat{\psi}_{g}^{\dagger} \hat{\mathbf{P}}^{+}\left(\mathbf{r}^{\prime}\right) \hat{\psi}_{g},
\end{aligned}
$$

where $\bar{\Delta}=\Delta_{e}-\Delta_{g}$.

In contrast to atoms with isotropic polarizability, the fixed orientation of the atomic dipoles of two-level atoms means that, in the low light intensity limit, we only need to consider the equation of motion for the scalar polarisation operator $\hat{P}^{+}$, which reduces in this limit to [from Eq. (26)]

$$
\begin{aligned}
\frac{d}{d t} \hat{P}^{+} & =(i \bar{\Delta}-\gamma) \hat{P}^{+}+i \xi \hat{\psi}_{g}^{\dagger} \hat{\psi}_{g} \hat{\mathbf{e}}^{*} \cdot \hat{\mathbf{D}}_{F}^{+} \\
& +i \xi \int d^{3} r^{\prime} \hat{\mathbf{e}}^{*} \cdot \mathrm{G}^{\prime}\left(\mathbf{r}-\mathbf{r}^{\prime}\right) \hat{\mathbf{e}} \hat{\psi}_{g}^{\dagger} \hat{P}^{+}\left(\mathbf{r}^{\prime}\right) \hat{\psi}_{g} .
\end{aligned}
$$

A two-level system can be realized, e.g., from a $J=0 \rightarrow$ $J^{\prime}=1$ transition (or from a cycling transition in the case of alkaline-metal atoms) by tuning all except one transition off resonance by a magnetic field.

In the low light intensity limit, it remains to solve Eqs. (31), (30), or (29), depending on the complexity of the system to be modeled. We show in the following section the degree to which these equations may be treated by stochastic techniques, and that they may be solved exactly in the case of atoms with a single electronic ground state. Later, in Sec. IV, we discuss the extension of these techniques to higher intensities, including the effects of saturation.

\section{STOCHASTIC CLASSICAL ELECTRODYNAMICS SIMULATIONS IN THE LOW LIGHT INTENSITY LIMIT}

\section{A. Exact solutions for atoms with a single ground level}

In the previous sections we introduced a quantum fieldtheoretical formalism for the coupled system of atoms and light. We will next use simple principles to derive equations of motion for stochastic classical electrodynamics simulations of the optical response. For atoms with a single electronic ground state, and in the limit of low light intensity (see Sec. II C 1), these stochastic simulations can provide exact solutions for the optical response of an ensemble of stationary atoms that in the full quantum field-theoretical analysis is represented by the hierarchy of equations of motion for correlation functions (App. B). In contrast, as we show in the following section, when generalizing this technique to treat atoms with multiple ground levels we must introduce a classical approximation for virtual fluctuations between different ground levels. 
In the low light intensity limit we shall show that the atoms can be treated as a collection of coupled linear oscillators whose positions are stochastically sampled from the appropriate spatial distribution. For each stochastic realization of a fixed set of atomic positions we solve the coupled electrodynamics for the atoms and light. The optical response of the ensemble is then given by averaging any derived quantities of interest, such as polarization, over many such realizations $[10,17,21]$. It was first shown in the case of 1D scalar electrodynamics in Ref. [10] that the stochastic classical electrodynamics simulations reproduce the full quantum field-theoretical representation for the hierarchy of equations of motion for light-induced correlation functions [Eq. (B8)]. We extend the simplified model of Ref. [10] to the 3D case of the $J=0 \rightarrow J^{\prime}=1$ transition in App. B, including the vector properties of the electromagnetic fields. Such coupled dipole model simulations treating atoms as classical, linear oscillators have been used to calculate the responses of two-level atoms [10, 17, 58], emitters with isotropic susceptibility [15, 19, 21, 48], or emitters with some spatial averaging over dipole orientations [14].

The procedure for calculating the response amounts to a Monte-Carlo integration of Eqs. (30) (for isotropic atoms) or (31) (for two-level atoms) in which a realization of discrete atomic positions $\left\{\mathbf{X}_{1}, \mathbf{X}_{2}, \ldots, \mathbf{X}_{N}\right\}$ is a set of random variables sampled from the joint probability distribution $\left|\Psi\left(\mathbf{r}_{1}, \mathbf{r}_{2}, \ldots, \mathbf{r}_{N}\right)\right|^{2}$, determined by the ground-state many-body wave function $\Psi\left(\mathbf{r}_{1}, \mathbf{r}_{2}, \ldots, \mathbf{r}_{N}\right)$ that describes the center-of-mass state of the atoms. If the atoms can be considered to be initially uncorrelated before the light enters the medium (for instance, in a classical vapor or in an ideal Bose-Einstein condensate), the sampling reduces to the simple procedure of independently sampling the position of atom $i$ from the atomic density distribution of the ensemble $\rho_{1}\left(\mathbf{r}_{i}\right)$ [as discussed further in App. B, see Eqs. (B9) and (B10)]. An explicit example of the position sampling is given later, in Sec. V, for a simple special case in a strongly correlated quantum system. Other examples include Fermi-Dirac statistics, which can be modeled using a Metropolis algorithm [10]. Quantum mechanical expectation values for the quantities of interest are then generated from an ensemble average over many stochastic realizations.

We will next present a simple procedure for deriving the classical electrodynamics equations for individual realizations of atomic positions $\left\{\mathbf{X}_{1}, \ldots, \mathbf{X}_{N}\right\}$. In order to obtain the equations governing the optical response of any particular stochastic realization, we take the expectation values of both sides of Eq. (30), conditioned on the $N$ atoms being at the positions $\mathbf{X}_{1}, \ldots, \mathbf{X}_{N}$. Since up until now we have been dealing with quantum field operators for the atoms, the procedure is tantamount to a hypothetical situation in which the atoms are physically pinned at fixed positions $\left\{\mathbf{X}_{1}, \ldots, \mathbf{X}_{N}\right\}$. The atomic density then becomes

$$
\left\langle\hat{\psi}_{g}^{\dagger} \hat{\psi}_{g}\right\rangle_{\left\{\mathbf{X}_{1}, \ldots, \mathbf{X}_{N}\right\}}=\sum_{j} \delta\left(\mathbf{r}-\mathbf{X}_{j}\right)
$$

where the subscript $\left\{\mathbf{X}_{1}, \ldots, \mathbf{X}_{N}\right\}$ indicates the conditioning of the expectation value. Similarly, the polarization density operator is

$$
\left\langle\hat{\mathbf{P}}^{+}(\mathbf{r})\right\rangle_{\left\{\mathbf{X}_{1}, \ldots, \mathbf{X}_{N}\right\}}=\mathcal{D} \sum_{j} \mathcal{P}^{(j)} \delta\left(\mathbf{r}-\mathbf{X}_{j}\right),
$$

where, for isotropic atoms, we define the normalized dipole amplitude $\mathcal{P}^{(j)}=\sum_{\sigma} \hat{\mathbf{e}}_{\sigma} \mathcal{P}_{0 \sigma}^{(j)}$ of atom $j$, conditioned on atoms being located at positions $\mathbf{X}_{1}, \ldots, \mathbf{X}_{N}$. Similarly, for two-level atoms, we define the conditional normalized dipole amplitude $\mathcal{P}^{(j)}=\hat{\mathbf{e}} \mathcal{P}^{(j)}$.

In the following, we will proceed explicitly with the derivation of the stochastic equations of motion for the isotropic case $J=0 \rightarrow J^{\prime}=1$. The expectation value for the density-polarization operator appearing under Eq. (30) is

$$
\begin{aligned}
& \left\langle\hat{\psi}_{g}^{\dagger}(\mathbf{r}) \hat{\mathbf{P}}^{+}\left(\mathbf{r}^{\prime}\right) \hat{\psi}_{g}(\mathbf{r})\right\rangle_{\left\{\mathbf{X}_{1}, \ldots, \mathbf{X}_{N}\right\}} \\
& =\mathcal{D} \sum_{j} \sum_{l \neq j} \mathcal{P}^{(l)} \delta\left(\mathbf{r}-\mathbf{X}_{j}\right) \delta\left(\mathbf{r}^{\prime}-\mathbf{X}_{l}\right) .
\end{aligned}
$$

Here we have explicitly avoided double counting of any atom by excluding the atom $j$ in the summation over $l$. The benefit of arranging all the atomic operators into normal order now becomes obvious. The normal ordering corresponds to a simple procedure of classical counting of atoms, in an analogy of photon counting theory in photon detection [59-61].

Substituting Eqs. (32), (33) and (34) into Eq. (30), we find that for isotropic atoms

$$
\begin{aligned}
& \frac{d}{d t} \sum_{j} \mathcal{P}^{(j)} \delta\left(\mathbf{r}-\mathbf{X}_{j}\right)=(i \bar{\Delta}-\gamma) \sum_{j} \mathcal{P}^{(j)} \delta\left(\mathbf{r}-\mathbf{X}_{j}\right)+i \frac{\xi}{\mathcal{D}} \sum_{j} \mathbf{D}_{F}^{+} \delta\left(\mathbf{r}-\mathbf{X}_{j}\right) \\
& \quad+i \xi \sum_{j} \sum_{l \neq j} \int d^{3} r^{\prime} \mathrm{G}^{\prime}\left(\mathbf{r}-\mathbf{r}^{\prime}\right) \mathcal{P}^{(l)} \delta\left(\mathbf{r}-\mathbf{X}_{j}\right) \delta\left(\mathbf{r}^{\prime}-\mathbf{X}_{l}\right),
\end{aligned}
$$


a multiplicative classical coherent field $\mathbf{D}_{F}^{+}$. Multiplying both sides of Eq. (35) by $\delta_{j, j^{\prime}}$, interchanging indices $j$ and $j^{\prime}$, and integrating over $\mathbf{r}$, we find the dynamics of the individual atomic dipole moments satisfy the coupled equations

$$
\begin{aligned}
& \frac{d}{d t} \mathcal{P}^{(j)}=(i \bar{\Delta}-\gamma) \mathcal{P}^{(j)}+i \frac{\xi}{\mathcal{D}} \mathbf{D}_{F}^{+}\left(\mathbf{X}_{j}\right) \\
& \quad+i \xi \sum_{l \neq j} \mathrm{G}^{\prime}\left(\mathbf{X}_{j}-\mathbf{X}_{l}\right) \mathcal{P}^{(l)}
\end{aligned}
$$

which may be used to study the time-dynamics of the polarization, e.g., by short resonant pulses. We may also obtain the steady-state solution to Eq. (36)

$$
\mathcal{P}^{(j)}=\frac{\alpha}{\mathcal{D}} \mathbf{D}_{F}^{+}\left(\mathbf{X}_{j}\right)+\alpha \sum_{l \neq j} \mathrm{G}^{\prime}\left(\mathbf{X}_{j}-\mathbf{X}_{l}\right) \mathcal{P}^{(l)},
$$

where the polarizability of the atom $\alpha$ is in this case isotropic, and defined by

$$
\alpha=-\frac{\mathcal{D}^{2}}{\hbar \epsilon_{0}(\bar{\Delta}+i \gamma)} .
$$

Both Eqs. (36) and (37) represent the coupled equations for atomic excitations in the presence of a driving incident light. The atoms are located at discrete positions $\left\{\mathbf{X}_{1}, \mathbf{X}_{2}, \ldots, \mathbf{X}_{N}\right\}$ and the interactions between the atoms are mediated by the scattered light field. As a consequence $\boldsymbol{P}^{(j)}$ depends not only on $\mathbf{X}_{j}$, but also on the position of all other atoms.

Each atom acts as a source of scattered dipole radiation providing a scattered field for (the positive frequency component) of the electric field $\epsilon_{0} \mathbf{E}_{S}^{(l)}(\mathbf{r})=\mathrm{G}^{\prime}(\mathbf{r}-$ $\left.\mathbf{X}_{l}\right) \mathcal{D} \mathcal{P}^{(l)}$, where the propagator is defined in Eq. (21) and gives the familiar expression for the electric field radiated by a dipole $\mathcal{D} \mathcal{P}^{(l)}$ situated at point $\mathbf{X}_{l}$. Each atom $j$ at position $\mathbf{X}_{j}$ in Eqs. (36) and (37) is therefore driven by the field $\mathbf{E}_{\text {ext }}^{+}\left(\mathbf{X}_{j}\right)$ that is the sum of the incident field $\mathbf{D}_{F}^{+}\left(\mathbf{X}_{j}\right)$ and the fields scattered from all $N-1$ other atoms in the system

$$
\epsilon_{0} \mathbf{E}_{\mathrm{ext}}^{+}\left(\mathbf{X}_{j}\right)=\mathbf{D}_{F}^{+}\left(\mathbf{X}_{j}\right)+\sum_{l \neq j} \epsilon_{0} \mathbf{E}_{S}^{(l)}\left(\mathbf{X}_{j}\right) .
$$

Focusing on the steady-state response (37), the scattered field then induces the polarization at the atom $j$ that is proportional to the polarizability $\alpha$ [Eq. (38)] $\mathcal{D} \mathcal{P}^{(j)}=\alpha \epsilon_{0} \mathbf{E}_{\text {ext }}^{+}\left(\mathbf{X}_{j}\right)$. Owing to the isotropic response of the atoms in case of the $J=0 \rightarrow J^{\prime}=1$ transition, the total electric field in the steady-state case (37) may be solved from

$$
\epsilon_{0} \mathbf{E}_{\text {ext }}^{+}\left(\mathbf{X}_{j}\right)=\mathbf{D}_{F}^{+}\left(\mathbf{X}_{j}\right)+\epsilon_{0} \alpha \sum_{l \neq j} \mathrm{G}^{\prime}\left(\mathbf{r}-\mathbf{X}_{l}\right) \mathbf{E}_{\text {ext }}^{+}\left(\mathbf{X}_{l}\right) \text {. }
$$

This linear set of equations can be solved to give the total electric field amplitude everywhere in space

$$
\epsilon_{0} \mathbf{E}^{+}(\mathbf{r})=\mathbf{D}_{F}^{+}(\mathbf{r})+\epsilon_{0} \alpha \sum_{j} \mathrm{G}^{\prime}\left(\mathbf{r}-\mathbf{X}_{j}\right) \mathbf{E}_{\mathrm{ext}}^{+}\left(\mathbf{X}_{j}\right)
$$

A convenient feature of the isotropic $J=0 \rightarrow J^{\prime}=1$ transition is that all the internal properties of the atoms in Eq. (40) are encapsulated in a single scalar quantity $\alpha$.

The response of the system at low light intensities for a single realization of atomic positions is equivalent to the response of a model of coupled harmonic oscillators at positions $\mathbf{X}_{1}, \ldots, \mathbf{X}_{N}$. The oscillators have dipole matrix elements $\mathcal{D}$, detuning $\bar{\Delta}$, and spontaneous linewidth $\gamma$ defined in Eq. (20).

In order to obtain the steady-state solution for the optical response of the atomic ensemble where the positions of the atoms are distributed according to some joint probability distribution $P\left(\mathbf{r}_{1}, \mathbf{r}_{2}, \ldots, \mathbf{r}_{N}\right)$, one then stochastically samples the atomic positions. For each stochastic realization of a set of discrete atomic positions $\left\{\mathbf{X}_{1}, \mathbf{X}_{2}, \ldots, \mathbf{X}_{N}\right\}$, one solves Eq. (40) for the electric field, or equivalently Eq. (37). With that solution, one calculates the observables of interest for each realization. The expectation value of those observables corresponds to the average over sufficiently many stochastic realizations. Dynamical calculations proceed analogously, calculating dynamical trajectories from Eq. (36) for individual realizations of atomic positions, and averaging over many trajectories.

Following the arguments of Ref. [10], we show in App. B that this sampling procedure generates the correct dynamics for many-body polarization-density correlation functions in the low light intensity limit. We note that, while we appear to neglect correlations between atoms in a single realization by using Eq. (34), App. B demonstrates the subsequent averaging over many stochastic realizations includes all relevant correlations for low light intensity coherent scattering.

While we focus on cold, homogeneously broadened ensembles of atoms, we note that thermal effects can also be approximated in this treatment. Thermal motion of the atoms introduces Doppler broadening of the resonance frequencies. This inhomogeneous broadening mechanism is accounted for by additionally stochastically sampling the resonance frequency detunings of each atom $\bar{\Delta}^{(j)}$ to reflect the distribution of thermal atomic velocities [21].

The two-level case can be treated analogously to the isotropic case. In contrast to atoms with isotropic polarizability, the atomic dipoles of two-level atoms have a fixed orientation $\hat{\mathbf{e}}$. As a consequence, the dipole moment of the atoms is proportional to the scalar $\mathcal{P}^{(j)} \equiv \hat{\mathbf{e}}^{*} \cdot \mathcal{P}^{(j)}$, and components of $\boldsymbol{P}^{(j)}$ orthogonal to $\hat{\mathbf{e}}$ are zero. By making the substitution $\mathcal{P}^{(j)} \rightarrow \hat{\mathbf{e}} \mathcal{P}^{(j)}$ into Eq. (36), we find that for a stochastic realization of two-level atomic positions, the dipole amplitudes evolve as

$$
\begin{aligned}
& \frac{d}{d t} \mathcal{P}^{(j)}=(i \bar{\Delta}-\gamma) \mathcal{P}^{(j)}+i \frac{\xi}{\mathcal{D}} \hat{\mathbf{e}}^{*} \cdot \mathbf{D}_{F}^{+}\left(\mathbf{X}_{j}\right) \\
& \quad+i \xi \sum_{l \neq j} \hat{\mathbf{e}}^{*} \cdot \mathrm{G}^{\prime}\left(\mathbf{X}_{j}-\mathbf{X}_{l}\right) \hat{\mathbf{e}} \mathcal{P}^{(l)} .
\end{aligned}
$$

Averaging over many such realizations generates the dynamics of the expectation value of the polarization, from 
which quantities of interest may be calculated. Alternatively, analogous arguments to those above can be used to directly solve for the steady state electric field.

\section{B. Approximate solutions for multilevel atoms}

In common experimental situations [22], the electronic ground state of the atoms may have nonzero angular momentum and multiple internal transitions participate in the scattering processes. In this section we consider the generalization of the stochastic classical electrodynamics simulations of Sec. III A to such cases. In contrast to single ground level atoms, the systems consisting of atoms with multiple ground levels support virtual fluctuations between Zeeman ground levels that can become correlated with the atomic polarization, even in the low light intensity limit. This added complexity manifests itself in the quantum field-theoretical representation for the coupled system of atoms and light that leads to the hierarchy of equations of motion for $\ell^{\text {th }}$ order correlation functions $(\ell=1, \ldots, N)$ that fully describe the ensemble's optical response [see Eqs. (B2)-(B5)]. As shown in App. B, rather than considering one vector-valued correlation function for each level of the dynamic hierarchy for isotropic atoms, the number of correlation functions involved in the optical response of multilevel atoms scales exponentially with the number of atoms in the system.

Physically, for single ground level atoms at the limit of low light intensity, the excitation of each atom can be described as a superposition of linear polarization amplitudes. There is therefore no additional internal level dynamics beyond the spatial positions of the atoms that could become correlated by the scattered light. A numerical treatment of the light-induced position correlations between the atoms is consequently sufficient to obtain the complete solution to the problem. However, for atoms possessing multiple ground levels the light may also generate nonclassical internal level correlations between different atoms. As a consequence, the classical electrodynamics simulations of charged harmonic oscillators at stochastically sampled positions that exactly reproduce the hierarchy for isotropic and two-level atoms (see Sec. III A) are not able to capture quantum fluctuations that may potentially arise in the case of multiple ground levels.

In this section, we show how to generalize the stochastic classical electrodynamics simulations of Sec. III A to approximate the cooperative response of an ensemble of atoms with multiple electronic ground states. We introduce a classical approximation where we include the recurrent scattering processes between the classical dipoles corresponding to the different atomic transitions, but nonclassical higher order correlations between internal levels that involve fluctuations between ground-state coherences of different levels and the polarization are factorized. We emphasize, however, that the single-particle expectation values of the ground state populations and coherences, themselves, are unaffected by these nonclassical correlation effects to first order in the electric field amplitude. An analogous factorization approximation of higher order internal atomic level correlations will also be employed in Sec. IV when we show how to simulate an ensemble's cooperative response outside the low light intensity limit. The classical electrodynamics approximation for atoms with multiple ground levels leads to a treatment where the atomic Zeeman levels, as well as the atomic positions, are stochastically sampled. This was first outlined and employed in Ref. [22] to model the experiments presented in that reference. We later show how to improve the approximation to account for pairwise correlations between virtual fluctuations of Zeeman states and atomic dipoles in Sec. III B 1.

As with isotropic and two-level atoms, we determine the optical response of the gas by stochastically sampling the positions of the atoms $\left\{\mathbf{X}_{1}, \ldots, \mathbf{X}_{N}\right\}$, and then determine the optical response for each such realization. Following the same general procedure established in Sec. III A, for each realization of atomic positions, we consider the hypothetical situation where atoms are pinned to the points $\left\{\mathbf{X}_{1}, \ldots, \mathbf{X}_{N}\right\}$. The dynamics of the atomic dipoles for each realization are then found by taking the expectation value of Eq. (29) conditioned on atoms being located at those points. For notational simplicity, we focus here on the common experimental situation where the atoms are initially (before the incident light enters the atomic gas) assumed to be in an incoherent mixture of ground levels $|g, \nu\rangle$, such that $\left\langle\hat{\psi}_{g \nu}^{\dagger} \hat{\psi}_{g \tau}\right\rangle=0$ if $\nu \neq \tau$. The treatment can be generalized also to the case where the initial Zeeman coherences between the different ground levels $\nu \neq \tau$ are nonvanishing and this will be briefly discussed towards the end of this section. In the limit of low light intensity, an initially incoherent mixture remains so at all times, and the relative populations of the different levels are invariant.

The ground-level densities conditioned on a single realization of discrete atomic positions can conveniently be represented as

$$
\left\langle\hat{\psi}_{g \nu}^{\dagger} \hat{\psi}_{g \nu}\right\rangle_{\left\{\mathbf{X}_{1}, \ldots, \mathbf{X}_{N}\right\}}=\sum_{j} f_{\nu}^{(j)} \delta\left(\mathbf{r}-\mathbf{X}_{j}\right)
$$

with relative occupations of the different ground levels $f_{\nu}^{(j)}\left(0 \leq f_{\nu}^{(j)} \leq 1\right.$ and $\left.\sum_{\nu} f_{\nu}^{(j)}=1\right)$, at position $\mathbf{X}_{j}$. In the limit of low light intensity these single-atom groundstate populations are constant and unaffected by light to first order in the electric field amplitude (see Sec. II C). Analogous to Eq. (33), we similarly obtain for a specific realization of the atomic positions the polarization

$$
\begin{aligned}
\left\langle\hat{\mathbf{P}}_{\nu \eta}^{+}\right\rangle_{\left\{\mathbf{X}_{1}, \ldots, \mathbf{X}_{N}\right\}} & =\mathcal{D} \sum_{\sigma} \hat{\mathbf{e}}_{\sigma} \mathcal{C}_{\nu, \eta}^{(\sigma)} \\
& \times \sum_{j} f_{\nu}^{(j)} \mathcal{P}_{\nu \eta}^{(j)}(t) \delta\left(\mathbf{r}-\mathbf{X}_{j}\right),
\end{aligned}
$$

where $\mathcal{P}_{\nu \eta}^{(j)}(t)$ denote the dynamic excitation amplitudes. 
The electric dipole for the atomic transition $|g, \nu\rangle \rightarrow$ $|e, \eta\rangle$ of atom $j$ is then given by $\mathcal{D} \hat{\mathbf{e}}_{\eta-\nu} \mathcal{C}_{\nu, \eta}^{(\eta-\nu)} f_{\nu}^{(j)} \mathcal{P}_{\nu \eta}^{(j)}$.

The expectation value for the density-polarization operator [appearing in the last term of Eq. (29)] involves the two-body correlation functions

$$
\begin{aligned}
& \left\langle\hat{\psi}_{g \nu}^{\dagger}(\mathbf{r}) \psi_{g \mu}^{\dagger}\left(\mathbf{r}^{\prime}\right) \hat{\psi}_{e \eta}\left(\mathbf{r}^{\prime}\right) \hat{\psi}_{g \tau}(\mathbf{r})\right\rangle_{\left\{\mathbf{X}_{1}, \ldots, \mathbf{X}_{N}\right\}} \\
& =\sum_{j} \sum_{l \neq j} \mathcal{P}_{\nu \tau ; \mu \eta}^{(j ; l)} \delta\left(\mathbf{r}-\mathbf{X}_{j}\right) \delta\left(\mathbf{r}^{\prime}-\mathbf{X}_{l}\right) .
\end{aligned}
$$

Here $\mathcal{P}_{\nu \tau ; \mu \eta}^{(j ; l)}$ denote two-atom internal level correlation functions. They characterize correlations involving a Zeeman coherence between the ground levels $\nu$ and $\tau$ of atom $j$ and the polarization amplitude for the transition $|g, \mu\rangle \rightarrow|e, \eta\rangle$ of another atom $l \neq j$. It might, at first, appear unnecessary to include such pair correlations that consists of a Zeeman coherence between different ground levels $\nu$ and $\tau$. We assumed an incoherent mixture for which $\left\langle\hat{\psi}_{g \nu}^{\dagger} \hat{\psi}_{g \tau}\right\rangle=0$ whenever $\nu \neq \tau-$ which then in the low light intensity remains valid at all times. However, while no single particle Zeeman coherences can be generated by optical scattering in an incoherent mixture in the low light intensity limit, we will show that light scattering can generate nonzero two-atom correlations $\mathcal{P}_{\nu \tau ; \mu \eta}^{(j ; l)}$, and we discuss the implications of this below.

Using these definitions by substituting Eqs. (43), (44), and (45) into Eq. (29), we obtain (for $f_{\nu}^{(j)} \neq 0$ )

$$
\begin{aligned}
& \frac{d}{d t} \sum_{j} \mathcal{P}_{\nu \eta}^{(j)} \delta\left(\mathbf{r}-\mathbf{X}_{j}\right)=\left[i \bar{\Delta}_{g \nu e \eta}-\gamma\right] \sum_{j} \mathcal{P}_{\nu \eta}^{(j)} \delta\left(\mathbf{r}-\mathbf{X}_{j}\right)+i \frac{\xi}{\mathcal{D}} \sum_{j} \mathcal{C}_{\nu, \eta}^{(\sigma)} \hat{\mathbf{e}}_{\sigma}^{*} \cdot \mathbf{D}_{F}^{+} \delta\left(\mathbf{r}-\mathbf{X}_{j}\right) \\
& \quad+i \xi \sum_{j} \sum_{l \neq j} \int d^{3} r^{\prime} \mathcal{C}_{\tau, \eta}^{(\sigma)} \hat{\mathbf{e}}_{\sigma}^{*} \cdot \mathbf{G}^{\prime}\left(\mathbf{r}-\mathbf{r}^{\prime}\right) \hat{\mathbf{e}}_{\varsigma} \mathcal{C}_{\mu, \zeta}^{(\varsigma)} \frac{\mathcal{P}_{\nu \tau ; \mu \zeta}^{(j ; l)}}{f_{\nu}^{(j)}} \delta\left(\mathbf{r}-\mathbf{X}_{j}\right) \delta\left(\mathbf{r}^{\prime}-\mathbf{X}_{l}\right)
\end{aligned}
$$

where $\bar{\Delta}_{g \nu e \eta}=\Delta_{e \eta}-\Delta_{g \nu}$, and repeated indices $\tau, \mu, \zeta$, $\sigma$ and $\varsigma$ are summed over.

As in Sec. III A, we can simplify Eq. (46) by multiplying both sides by $\delta_{j, j^{\prime}}$, interchanging the indices $j$ and $j^{\prime}$, and integrating over $\mathbf{r}$, thus obtaining

$$
\begin{aligned}
& \frac{d}{d t} \mathcal{P}_{\nu \eta}^{(j)}=\left(i \bar{\Delta}_{g \nu e \eta}-\gamma\right) \mathcal{P}_{\nu \eta}^{(j)}+i \frac{\xi}{\mathcal{D}} \mathcal{C}_{\nu, \eta}^{(\sigma)} \hat{\mathbf{e}}_{\sigma}^{*} \cdot \mathbf{D}_{F}^{+}\left(\mathbf{X}_{j}\right) \\
& \quad+i \xi \sum_{l \neq j} \mathcal{C}_{\tau, \eta}^{(\sigma)} \hat{\mathbf{e}}_{\sigma}^{*} \cdot \mathrm{G}^{\prime}\left(\mathbf{X}_{j}-\mathbf{X}_{l}\right) \hat{\mathbf{e}}_{\varsigma} \mathcal{C}_{\mu, \zeta}^{(\varsigma)} \frac{\mathcal{P}_{\nu \tau ; \mu \zeta}^{(j ; l)}}{f_{\nu}^{(j)}}
\end{aligned}
$$

In the absence of magnetic Zeeman splitting $(B=0)$, the steady state polarization amplitudes for a specific atom $j$ become

$$
\begin{aligned}
\mathcal{P}_{\nu \eta}^{(j)} & =\frac{\alpha_{\nu \eta}}{\mathcal{D}} \mathcal{C}_{\nu, \eta}^{(\sigma)} \hat{\mathbf{e}}_{\sigma}^{*} \cdot \mathbf{D}_{F}^{+}\left(\mathbf{X}_{j}\right) \\
& +\alpha_{\nu \eta} \sum_{l \neq j} \mathcal{C}_{\tau, \eta}^{(\sigma)} \hat{\mathbf{e}}_{\sigma}^{*} \cdot \mathbf{G}^{\prime}\left(\mathbf{X}_{j}-\mathbf{X}_{l}\right) \hat{\mathbf{e}}_{\varsigma} \mathcal{C}_{\mu, \zeta}^{(\varsigma)} \frac{\mathcal{P}_{\nu \tau ; \mu \zeta}^{(j ; l)}}{f_{\nu}^{(j)}}
\end{aligned}
$$

In Eqs. (47) and (48), repeated indices $\tau, \mu, \zeta, \sigma$, and $\varsigma$ are summed over. The atomic polarizability is now anisotropic

$$
\alpha_{\nu \eta}=-\frac{\mathcal{D}^{2}}{\hbar \epsilon_{0}\left(\bar{\Delta}_{g \nu e \eta}+i \gamma\right)} .
$$

We have now removed the spatial correlations of the atoms by considering the atoms at fixed positions. In the simulations this represents an individual stochastic realization when the atomic configuration has been sampled from the joint probability distribution of the atomic positions. However, due to the multiple internal ground levels, correlations between the levels may still exist. Generally, to calculate the optical response, one must therefore determine the dynamics of the two-atom internal level correlations $\mathcal{P}_{\nu \tau ; \mu \zeta}^{(j ; l)}$. We will see later in Sec. III B 1 that the internal level pair correlations depend on three-atom internal level correlations, and so on. The equations of motion for these internal level correlation functions may be derived from the quantum field-theoretical hierarchy of equations of motion for multilevel density-polarization correlation functions presented in App. B [see Eqs. (B2)(B5)] when the atoms are pinned at the stochastically sampled points $\left\{\mathbf{X}_{1}, \ldots, \mathbf{X}_{N}\right\}$. In contrast to the single ground level case (as discussed in Sec. III A), for multiple ground levels the full field-theoretical description [Eq. (B5)] cannot be solved exactly by tracking only single-atom internal level correlations for each realization of atomic positions. Rather, $\mathcal{P}_{\nu \tau ; \mu \zeta}^{(j ; l)}$ does not factorize into single-atom internal level correlations and describes pair correlations between internal levels, involving virtual fluctuations between ground-state coherences of different levels and the polarization. We will briefly discuss the dynamics of $\mathcal{P}_{\nu \tau ; \mu \zeta}^{(j ; l)}$ in Sec. III B 1

Here we introduce a computationally efficient classical approximation where the virtual fluctuations between ground-state coherences of different levels and the polarization are neglected. We assume that the correlations $\mathcal{P}_{\nu \tau ; \mu \zeta}^{(j ; l)}$ for the atoms $j$ and $l$ factorize,

$$
\mathcal{P}_{\nu \tau ; \mu \eta}^{(j ; l)} \simeq \delta_{\nu \tau} f_{\nu}^{(j)} f_{\mu}^{(l)} \mathcal{P}_{\mu \eta}^{(l)}
$$


where the Kronecker delta $\delta_{\nu \tau}$ appears due to the assumption of an incoherent mixture of ground levels. With this simplification, Eqs. (47) and (48) each become a closed set of linear equations resembling the electrodynamic response of a set of classical linear oscillators. These can be solved for each set of fixed atomic positions. Ensemble-averaging over many stochastic realizations then generates light-induced correlations between the atoms.

For a single realization, the temporal evolution and the steady state of the atomic polarization can be inferred from Eqs. (47) and (48) [with the approximation of Eq. (50)], respectively. For each occupied Zeeman ground state (i.e., levels $|g, \nu\rangle$ for which $f_{\nu}^{(j)} \neq 0$ ), the amplitudes $\mathcal{P}_{\nu, \nu+\sigma}^{(j)}(\sigma=0, \pm 1)$ potentially contribute to the dipole moment of atom $j$. At first glance, one might expect that for every atom, one would have to track three amplitudes for every occupied Zeeman ground level. A numerically efficient alternative to this approach, however, is to stochastically sample ground level occupations for every realization of atomic positions. Specifically, for each realization, one randomly samples the atomic positions $\left\{\mathbf{X}_{1}, \ldots, \mathbf{X}_{N}\right\}$, drawn from the many-body joint probability distribution. Then, for each atom $j=1, \ldots, N$, one stochastically samples the atom's initial ground level $\left|g, M_{j}\right\rangle$, where the probability of choosing the Zeeman state $\left\{M_{1}, \ldots, M_{N}\right\}$ is governed by the populations $f_{\nu}^{(j)}$, which can be obtained from the initial many-body distribution. For each stochastic realization, each atom is then described by only three amplitudes $\mathcal{P}_{M_{j}, M_{j}+\sigma}^{(j)}$ $(\sigma=0, \pm 1$ ), which have the steady-state solutions (with the repeated indices $\sigma, \varsigma$, and $\zeta$ summed over)

$$
\begin{aligned}
& \mathcal{P}_{M_{j} \eta}^{(j)}=\frac{\alpha_{M_{j} \eta}}{\mathcal{D}} \mathcal{C}_{M_{j}, \eta}^{(\sigma)} \hat{\mathbf{e}}_{\sigma}^{*} \cdot \mathbf{D}_{F}^{+}\left(\mathbf{X}_{j}\right) \\
& +\alpha_{M_{j} \eta} \sum_{l \neq j} \mathcal{C}_{M_{j}, \eta}^{(\sigma)} \hat{\mathbf{e}}_{\sigma}^{*} \cdot \mathbf{G}^{\prime}\left(\mathbf{X}_{j}-\mathbf{X}_{l}\right) \hat{\mathbf{e}}_{\varsigma} \mathcal{C}_{M_{l}, \zeta}^{(\varsigma)} \mathcal{P}_{M_{l} \zeta}^{(l)}
\end{aligned}
$$

From the solution of the steady-state or timedependent amplitudes for each realization, one can calculate the scattered electric field and other related observables. We can then write the total electric field as the sum of the incident field and the fields scattered from each atom in the ensemble, which for a single realization of atomic positions and Zeeman levels is given by

$$
\epsilon_{0} \mathbf{E}(\mathbf{r}, t)=\mathbf{D}_{F}^{+}(\mathbf{r}, t)+\mathcal{D} \sum_{j} \mathrm{G}^{\prime}\left(\mathbf{r}-\mathbf{X}_{j}\right) \hat{\mathbf{e}}_{\sigma} \mathcal{C}_{M_{j}, \eta}^{(\sigma)} \mathcal{P}_{M_{j} \eta}^{(j)}
$$

where repeated indices $\sigma$ and $\eta$ are summed over. [The single ground level analogue of Eq. (52) for a $J=0 \rightarrow$ $J^{\prime}=1$ transition is given by Eq. (41).] Subsequent averaging over a large enough ensemble of realizations of positions and Zeeman states provides the expectation values of the scattered electric field and other observables of interest. The multilevel classical electrodynamics simulations have recently been implemented to study the pulsed excitations of ${ }^{87} \mathrm{Rb}$ atoms in the $F=2$ ground- state manifold for fluorescence and coherent light transmission $[22,49,50]$.

Though the classical approximation of Eq. (50) neglects the quantum virtual fluctuations between different Zeeman ground levels, as mentioned earlier, averaging over many stochastic realizations of positions and Zeeman states still produces correlations between the polarization, density, and atomic Zeeman states. In particular, as in Sec. III A, recurrent scattering processes are included in this treatment. However, in contrast to the case of a single ground level system, due to the approximation of Eq. (50) we no longer exactly reproduce the dynamics of the full quantum field-theoretical representation of the optical response that is expressed by the hierarchy of equations for the correlation functions given in App. B. A similar approximation for two-level atoms outside the low light intensity limit will be discussed in Sec. IV A.

We have focused here on describing the response of atomic ensembles that initially - before the light enters the sample - are prepared in an incoherent mixture of ground levels. Ground level coherences could be included by replacing Eq. (43) by the more general expression

$$
\left\langle\hat{\psi}_{g \nu}^{\dagger} \hat{\psi}_{g \tau}\right\rangle_{\left\{\mathbf{x}_{1}, \ldots, \mathbf{X}_{N}\right\}}=\sum_{j} \varrho_{\nu \tau}^{(j)} \delta\left(\mathbf{r}-\mathbf{X}_{j}\right) .
$$

Here $\varrho_{\nu \tau}^{(j)}$ describe coherences between internal ground levels $\nu$ and $\tau$ for atom $j$ in a single realisation of atomic positions. In the limit of low light intensity, such coherences are invariant with the exception of a phase rotation resulting from any energy splitting of the ground levels. An analogous treatment to that presented above then leads to generalized equations of motion for a single stochastic realization. Later, in Sec. IV B, we show explicitly how ground level coherences may be taken into account outside of the low light intensity limit, where one allows for saturation and dynamics between all Zeeman levels.

\section{Incorporating virtual Zeeman fluctuations}

The stochastic technique described above removes the spatial correlations in each individual stochastic realization and the electrodynamics can be solved for a fixed set of atomic positions. Generally, in the presence of multiple internal ground levels, there is additional internal-level dynamics beyond the spatial positions of the atoms that can in principle lead to nonclassical correlations between the different atoms mediated by the scattered light. The classical approximation, we introduced in the previous section, assumes that the two-atom internal level correlation functions $\mathcal{P}_{\nu \tau ; \mu \eta}^{(j ; l)}$ can be factorized according to Eq. (50), leading to a coupled multilevel dipole model. In order to incorporate quantum effects beyond the classical model, we must track the virtual fluctuations between different ground Zeeman states in $\mathcal{P}_{\nu \tau ; \mu \eta}^{(j ; l)}$ in each realiza- 
tion of atomic positions. We now discuss how to track this quantity, accounting for the possibility that correlations may form between these virtual fluctuations and atomic dipoles.

We treat the two-atom internal level correlation functions on which the single-atom amplitudes depend [see Eq. (47)] in a way similar to the method we employed to derive the dynamics for $\mathcal{P}_{\nu \eta}^{(j)}$. Using Eqs. (19) and (22), one can write the equation of motion, to first order in the electric field, of the products of field operators appear- ing in Eq. (29). For a general initial many-body atomic wave function, the polarization can be found by solving the hierarchy of equations of motion for the correlation functions presented in App. B [7]. For a specific realization of atomic positions, one expresses the correlation functions subject to the atoms being at those positions $\left\{\mathbf{X}_{1}, \ldots, \mathbf{X}_{N}\right\}$. Doing so results in a two-atom ground state correlation function that interacts with the driving field. We write the ground-state pair correlation function

$$
\left\langle\hat{\psi}_{g \nu}^{\dagger}\left(\mathbf{r}_{1}\right) \hat{\psi}_{g \mu}^{\dagger}\left(\mathbf{r}_{2}\right) \hat{\psi}_{g \eta}\left(\mathbf{r}_{2}\right) \hat{\psi}_{g \tau}\left(\mathbf{r}_{1}\right)\right\rangle_{\left\{\mathbf{X}_{1}, \ldots, \mathbf{X}_{N}\right\}}=\sum_{j_{1}} \sum_{j_{2} \neq j_{1}} \varrho_{\nu \tau, \mu \eta}^{\left(j_{1}, j_{2}\right)} \delta\left(\mathbf{r}_{1}-\mathbf{X}_{1}\right) \delta\left(\mathbf{r}_{2}-\mathbf{X}_{2}\right)
$$

where $\varrho_{\nu \tau, \mu \eta}^{\left(j_{1}, j_{2}\right)}$ is the internal ground-level pair correlation function determined by the initial many-body wavefunction before the incident light enters the gas. (To first order in the electric field amplitude, $\varrho_{\nu \tau, \mu \eta}^{\left(j_{1}, j_{2}\right)}$ is invariant up to a phase rotation caused by any Zeeman splitting of the ground level.) We express the three-atom correlation function resulting from light scattering from atoms not at $\mathbf{r}_{1}$ or $\mathbf{r}_{2}$ as

$$
\begin{aligned}
& \left\langle\hat{\psi}_{g \nu_{1}}^{\dagger}\left(\mathbf{r}_{1}\right) \hat{\psi}_{g \nu_{2}}^{\dagger}\left(\mathbf{r}_{2}\right) \hat{\psi}_{g \nu_{3}}^{\dagger}\left(\mathbf{r}_{3}\right) \hat{\psi}_{e \eta_{3}}\left(\mathbf{r}_{3}\right) \hat{\psi}_{g \tau_{2}}\left(\mathbf{r}_{2}\right) \hat{\psi}_{g \tau_{1}}\left(\mathbf{r}_{1}\right)\right\rangle_{\left\{\mathbf{X}_{1}, \ldots, \mathbf{X}_{N}\right\}} \\
& =\sum_{\left(j_{1}, j_{2}, j_{3}\right)} \mathcal{P}_{\nu_{1} \tau_{1}, \nu_{2} \tau_{2} ; \nu_{3} \eta_{3}}^{\left(j_{1}, j_{2} ; j_{3}\right)} \delta\left(\mathbf{r}_{1}-\mathbf{X}_{1}\right) \delta\left(\mathbf{r}_{2}-\mathbf{X}_{2}\right) \delta\left(\mathbf{r}_{3}-\mathbf{X}_{3}\right) .
\end{aligned}
$$

where $\mathcal{P}_{\nu_{1} \tau_{1}, \nu_{2} \tau_{2} ; \nu_{3} \eta_{3}}^{\left(j_{1}, j_{2} ; j_{3}\right)}$ is the three-atom internal level correlation function involving ground state Zeeman coherences of atoms $j_{1}$ and $j_{2}$ and the dipole amplitude of atom $j_{3}$, at the positions $\mathbf{X}_{1}, \mathbf{X}_{2}$, and $\mathbf{X}_{3}$, respectively. The summation is over all permutations of distinct indices $\left(j_{1}, j_{2}, j_{3}\right)$ in the set $\{j=1 \ldots N\}$.

Here, as before, we assume the atoms are initially, in the absence of light, in an incoherent mixture of Zeeman levels. Furthermore, for simplicity, in the following we will consider an atomic system that does not exhibit correlations in the absence of the incident light, so that we can write in Eq. (54) the correlation function

$$
\varrho_{\nu \tau, \mu \eta}^{\left(j_{1}, j_{2}\right)}=f_{\nu}^{\left(j_{1}\right)} f_{\mu}^{\left(j_{2}\right)} \delta_{\nu \tau} \delta_{\mu \eta}
$$

For a specific realization of atomic positions, the twoatom amplitudes evolve according to [Eq. (B5)]

$$
\begin{aligned}
& {\left[\frac{d}{d t}-i\left(\bar{\Delta}_{g \mu e \eta}+\bar{\Delta}_{g \nu g \tau}\right)+\gamma\right] \mathcal{P}_{\nu \tau ; \mu \eta}^{\left(j_{1} ; j_{2}\right)}} \\
& =i \frac{\xi}{\mathcal{D}} f_{\nu}^{\left(j_{1}\right)} f_{\mu}^{\left(j_{2}\right)} \delta_{\nu \tau} \mathcal{C}_{\mu, \eta}^{(\sigma)} \hat{\mathbf{e}}_{\sigma}^{*} \cdot \mathbf{D}_{F}^{+}\left(\mathbf{X}_{j_{2}}\right) \\
& +i \xi \mathcal{C}_{\beta, \eta}^{(\sigma)} \hat{\mathbf{e}}_{\sigma}^{*} \cdot \mathrm{G}^{\prime}\left(\mathbf{X}_{j_{2}}-\mathbf{X}_{j_{1}}\right) \hat{\mathbf{e}}_{\varsigma} \mathcal{C}_{\tau, \zeta}^{(\varsigma)} \mathcal{P}_{\mu \beta ; \nu \zeta}^{\left(j_{2} ; j_{1}\right)} \\
& +i \xi \sum_{j_{3} \notin\left\{j_{1}, j_{2}\right\}} \mathcal{C}_{\beta, \eta}^{(\sigma)} \hat{\mathbf{e}}_{\sigma}^{*} \cdot \mathrm{G}^{\prime}\left(\mathbf{X}_{j_{2}}-\mathbf{X}_{j_{3}}\right) \hat{\mathbf{e}}_{\zeta} \mathcal{C}_{\epsilon, \zeta}^{(\varsigma)} \mathcal{P}_{\nu \tau, \mu \beta ; \epsilon \zeta}^{\left(j_{1}, j_{2} ; j_{3}\right)}
\end{aligned}
$$

where repeated indices $\beta, \epsilon, \zeta, \sigma$ and $\varsigma$ are summed over. The first term on the right-hand-side of Eq. (57) repre- sents driving of the two-atom internal level correlation function by the incident field. The second term on the right-hand-side is responsible for the recurrent scattering processes where an atomic polarization excitation is repeatedly exchanged between atoms $j_{1}$ and $j_{2}$ that are fixed at the positions $\mathbf{X}_{j_{1}}$ and $\mathbf{X}_{j_{2}}$, respectively. In the second term we can identify two types of dynamics: (i) recurrent scattering events for which $\nu=\tau$ and $\mu=\beta$, indicating that an excitation is exchanged between a groundstate atom and a polarization; (ii) processes in which one or both of the following are true: $\nu \neq \tau$ and $\mu \neq \beta$.

The case (i) represents classical electrodynamics of coupled dipoles, and in App. $\mathrm{C}$ we show that these processes are already incorporated in the multilevel classical approximation of Eq. (50). The case (ii) describes a virtual scattering process between atoms $j_{1}$ and $j_{2}$. This virtual scattering term is no longer classical because, even when the atoms are initially in an incoherent superposition of Zeeman levels, the photon exchange process can generate correlations arising from virtual fluctuations between Zeeman states in one atom and the dipole amplitude of another. Roughly speaking, as the dipole excitation is transferred from one atom to another, the virtual emission and reabsorption process can leave behind fluctuations between Zeeman levels in the emitting atom that are correlated with the absorbing atom's dipole amplitude. We emphasize, however, that single-atom ground state populations and coherences, themselves, are unaf- 
fected by virtual photon exchanges to first order in the electric field amplitude.

The last term of Eq. (57) is the driving of a two-atom internal level correlation function by three-atom internal level correlation functions that represent the dipole amplitude of an atom $j_{3} \neq j_{1}, j_{2}$ and atoms $j_{1}$ and $j_{2}$ being in the ground level. In principle, to solve the optical response of the cloud exactly, one would need to find the dynamics of the three-atom internal level correlation functions. This would, in turn, require one to solve the dynamics of four-atom internal level correlation functions, and so on. The result is a hierarchy of equations for the internal states of discrete emitters at fixed positions. This hierarchy is reminiscent of the full density-polarization correlation function hierarchy presented in App. B [7] when the atoms are at fixed positions $\left\{\mathbf{X}_{1}, \ldots, \mathbf{X}_{N}\right\}$.

In App. $\mathrm{C}$ we show that if we only consider the case (i) of Eq. (57), for which $\nu=\tau$ and $\mu=\beta$, and the corresponding hierarchy of equations for the internal level correlation functions with only the analogous diagonal terms $\mathcal{P}_{\nu_{1} \nu_{1}, \ldots, \nu_{\ell-1} \nu_{\ell-1} ; \nu_{\ell} \zeta}^{\left(j_{1}, \ldots, j_{\ell-1} ; j_{\ell}\right)}$ included, we can solve the entire hierarchy by the classical electrodynamics simulations of the previous section. For the $\ell$ th order correlation functions we substitute

$$
\mathcal{P}_{\nu_{1} \nu_{1}, \ldots, \nu_{\ell-1} \nu_{\ell-1} ; \nu_{\ell} \zeta}^{\left(j_{1}, \ldots, j_{\ell-1} ; j_{\ell}\right)}=f_{\nu_{1}}^{\left(j_{1}\right)} \ldots f_{\nu_{\ell}}^{\left(j_{\ell}\right)} \mathcal{P}_{\nu_{\ell} \zeta}^{\left(j_{\ell}\right)}
$$

With this substitution, the entire hierarchy can be reduced to the coupled time-dependent equations of multilevel dipoles [Eq. (47) with Eq. (50)]

$$
\begin{aligned}
& \frac{d}{d t} \mathcal{P}_{\mu \eta}^{(j)}=\left(i \bar{\Delta}_{g \mu e \eta}-\gamma\right) \mathcal{P}_{\mu \eta}^{(j)}+i \frac{\xi}{\mathcal{D}} \mathcal{C}_{\mu, \eta}^{(\sigma)} \hat{\mathbf{e}}_{\sigma}^{*} \cdot \mathbf{D}_{F}^{+}\left(\mathbf{X}_{j}\right) \\
& \quad+i \xi \sum_{l \neq j} \mathcal{C}_{\mu, \eta}^{(\sigma)} \hat{\mathbf{e}}_{\sigma}^{*} \cdot \mathbf{G}^{\prime}\left(\mathbf{X}_{j}-\mathbf{X}_{l}\right) \hat{\mathbf{e}}_{\varsigma} \mathcal{C}_{\epsilon, \zeta}^{(\varsigma)} f_{\epsilon}^{(l)} \mathcal{P}_{\epsilon \zeta}^{(l)}
\end{aligned}
$$

However, in order to solve the full quantum response we also need to include the terms of case (ii) of Eq. (57). To make such a solution tractable for a system of many atoms, we can truncate the hierarchy at the level of two-atom amplitudes. To do this, we approximate $\mathcal{P}_{\nu_{1} \tau_{1}, \nu_{2} \tau_{2} ; \nu_{3} \eta_{3}}^{\left(j_{1}, j_{2} ; j_{3}\right)}$ as a function of one and two atom quantities. This function is not unique, and the optimal choice may depend on the particular physical system. For example one could write, for an incoherent mixture

$$
\mathcal{P}_{\nu_{1} \tau_{1}, \nu_{2} \tau_{2} ; \nu_{3} \eta_{3}}^{\left(j_{1}, j_{2} ; j_{3}\right)} \approx f_{\nu_{1}}^{\left(j_{1}\right)} \delta_{\nu_{1} \tau_{1}} \mathcal{P}_{\nu_{2} \eta_{2} ; \nu_{3} \eta_{3}}^{\left(j_{2} ; j_{3}\right)}+f_{\nu_{2}}^{\left(j_{2}\right)} \delta_{\nu_{2} \tau_{2}} \mathcal{P}_{\nu_{1} \tau_{1} ; \nu_{3} \eta_{3}}^{\left(j_{1} ; j_{3}\right)}-f_{\nu_{1}}^{\left(j_{1}\right)} f_{\nu_{2}}^{\left(j_{2}\right)} \delta_{\nu_{1} \tau_{1}} \delta_{\nu_{2} \tau_{2}} \mathcal{P}_{\nu_{3} \eta_{3}}^{\left(j_{3}\right)}
$$

Substituting Eq. (60) into Eq. (57), one finds that Eq. (57) forms a closed system of equations for $\mathcal{P}_{\nu \tau ; \mu \eta}^{\left(j_{1} ; j_{2}\right)}$. The solution of these equations, in conjunction with Eq. (47), gives the optical response for a single realization of atomic positions $\left\{\mathbf{X}_{1}, \ldots, \mathbf{X}_{N}\right\}$. Ensemble averaging over many realizations yields the optical response of the gas.

\section{STOCHASTIC ELECTRODYNAMICS SIMULATIONS OF THE OPTICAL RESPONSE OF SATURATED ATOMS}

The full quantum field-theoretical representation for the cooperative response of the ensemble of two-level atoms in the low light intensity limit can be written as a hierarchy of $N$ equations of motion for correlation functions - with each correlation function involving only a single excited-state field operator [given by the two-level equivalent of Eq. (B8)]. Outside of the low light intensity limit this is no longer possible; an equivalent calculation requires one to consider equations for all atomic correlation functions. Even in an ensemble of two-level atoms, one must track all $2^{n} n$-body correlation functions for every $n<N$; multiple internal levels further increase the complexity. Consequently, accounting for saturation makes the solution of the optical response significantly more difficult. Fortunately, the classical electrodynamics simulation techniques used in the previous section can be extended to include the effects of saturation. When the excited-state populations are not negligible we explicitly keep track of the internal level dynamics of the atoms and introduce an approximation that is therefore semiclassical in nature. We also incorporate the light-induced correlations between the different atoms and different levels that depend on the spatial distribution of the atoms, but ignore quantum-mechanical fluctuations between the different levels [by means of factorizing many-body internal level correlation functions for discrete position realizations, in a manner analogous to that of Sec. IIIB]. We first discuss the simple case of two-level atoms before generalizing to the full description of multilevel atoms.

\section{A. Two-level atoms}

As in the low light intensity case, we seek the solution of the equations governing the optical response of two-level atoms in the presence of saturation [Eqs. (26) and (28)] by deriving equations of motion for the electrodynamics of $N$ atoms located at a set of discrete positions $\left\{\mathbf{X}_{1}, \ldots, \mathbf{X}_{N}\right\}$. The procedure removes the spatial correlations in each individual stochastic realization and the electrodynamics can be solved for a fixed set of atomic 
positions. The solution to the optical response of an atomic ensemble with distributed atomic positions is then obtained by means of averaging over many such realizations; this restores the correct spatial correlations when the positions in each realization are sampled from an appropriate $N$-body joint probability distribution function for the system.

In order to derive the equations of motion for pointlike scatterers at fixed discrete positions $\left\{\mathbf{X}_{1}, \ldots, \mathbf{X}_{N}\right\}$ for individual stochastic realizations, we use a similar mathematical procedure as in the low light intensity case of Sec. III A. We take the expectation values with respect to the center-of-mass coordinates of the atoms of both sides of Eqs. (26) and (28) conditioned on the $N$ atoms being at the positions $\mathbf{X}_{1}, \ldots, \mathbf{X}_{N}$, as if the atoms were in a hypothetical physical system pinned at fixed positions. However, since here we incorporate the saturation of the atoms, there also exists additional internal-level dynamics beyond the spatial positions of the atoms that can become correlated by the scattered light. The system therefore no longer resembles the classical electrodynamics of a set of linear harmonic oscillators and the internal states of different atoms can in principle be nonclassically correlated. The conditioned expectation values of onebody operators can be expressed in terms of one-body internal-level density matrix elements [68]

$$
\begin{aligned}
\left\langle\hat{\psi}_{e}^{\dagger} \hat{\psi}_{e}\right\rangle_{\left\{\mathbf{X}_{1}, \ldots, \mathbf{X}_{N}\right\}} & =\sum_{j} \rho_{e e}^{(j)} \delta\left(\mathbf{r}-\mathbf{X}_{j}\right), \\
\left\langle\hat{\psi}_{g}^{\dagger} \hat{\psi}_{g}\right\rangle_{\left\{\mathbf{X}_{1}, \ldots, \mathbf{X}_{N}\right\}} & =\sum_{j} \rho_{g g}^{(j)} \delta\left(\mathbf{r}-\mathbf{X}_{j}\right), \\
\left\langle\hat{\mathbf{P}}^{+}(\mathbf{r})\right\rangle_{\left\{\mathbf{X}_{1}, \ldots, \mathbf{X}_{N}\right\}} & =\mathcal{D} \hat{\mathbf{e}} \sum_{j} \rho_{g e}^{(j)} \delta\left(\mathbf{r}-\mathbf{X}_{j}\right),
\end{aligned}
$$

where we then require $\rho_{e e}^{(j)}+\rho_{g g}^{(j)}=1$ for the conservation of the atom population. In Eq. (63) we have explicitly written the fixed orientation $\hat{\mathbf{e}}$ of the atomic dipoles for two-level atoms. The two-body density-polarization correlations from Eqs. (26) and (28) for a single realization can similarly be represented by two-body internal-level density matrix elements

$$
\begin{aligned}
\left\langle\hat{\psi}_{a}^{\dagger}(\mathbf{r}) \hat{\psi}_{b}^{\dagger}\left(\mathbf{r}^{\prime}\right) \hat{\psi}_{c}\left(\mathbf{r}^{\prime}\right) \hat{\psi}_{d}(\mathbf{r})\right\rangle_{\left\{\mathbf{X}_{1}, \ldots, \mathbf{X}_{N}\right\}} & \\
= & \sum_{\substack{j, l=1 \\
j \neq l}}^{N} \rho_{a d ; b c}^{(j, l)} \delta\left(\mathbf{r}-\mathbf{X}_{j}\right) \delta\left(\mathbf{r}^{\prime}-\mathbf{X}_{l}\right) .
\end{aligned}
$$

The matrix elements $\rho_{a d ; b c}^{(j, l)}(a, b, c, d=g, e)$ represent internal level pair correlations between atoms at the fixed points $\mathbf{X}_{j}$ and $\mathbf{X}_{l}$. While, in the limit of low light intensity, only correlations involving at most one excited state operator were non-negligible, at arbitrary intensities we must include all 16 possible internal level pair correlations. Coupled equations of motion for such pair correlations can be obtained, following the method we earlier used to obtain Eq. (57), which depend in turn upon three-body internal level correlations, and so on. To develop a computationally efficient numerical method, we introduce a semiclassical approximation for the internal level pair correlations in a single realization of discrete atomic positions by factorizing them between different atoms, analogously to the approximation introduced in Sec. III B. For example, we take

$$
\rho_{g g ; g e}^{(j, l)} \simeq \rho_{g g}^{(j)} \rho_{g e}^{(l)}
$$

In fact, we made an analogous substitution earlier, in Sec. III A, for two-level atoms in the limit of low light intensity (or indeed, any atoms with a single electronic ground level). In that limit, it was shown in Ref. [10] and in App. B that the subsequent averaging over an ensemble of many such discrete positions realizations reproduces the dynamics of the full quantum field-theoretical representation of the correlation functions that govern the optical response. Within statistical accuracy, the stochastic electrodynamics simulations therefore give the exact optical response for an ensemble of stationary twolevel atoms in this limit. However, the argument of App. B relies on the linearity of the the model of coupled linear oscillators that results for each realization.

In contrast, in the saturated case the dynamics inherently involves the excited level and therefore constitutes a multilevel system. This semiclassical approximation stems from an analogous substitution to that which led to the classical approximation of the pair correlations for atoms with multiple ground levels in the low intensity case [Sec. III B]. Analogously to the multilevel low light intensity case, the scattered light may induce nonclassical correlations between the internal levels of different atoms that no longer can be captured by classical stochastic sampling and the electrodynamics of one-body operators, such as $\rho_{g e}^{(j)}$, by the factorization approximation Eq. (65). Specifically in this case, the nonlinear saturation at higher intensities prevents a similar solution to that of the low light intensity two-level case, and indeed the factorization of two-body internal level correlations introduces a semiclassical approximation.[69] In essence, we neglect internal state correlations between atoms in a single realization for quantities similar to $\left\langle\hat{\mathbf{P}}^{+}(\mathbf{r}) \hat{\mathbf{P}}^{-}\left(\mathbf{r}^{\prime}\right)\right\rangle$, and averaging over many realizations does not fully restore all the correlations. Terms like $\left\langle\hat{\mathbf{P}}^{+}(\mathbf{r}) \hat{\mathbf{P}}^{-}\left(\mathbf{r}^{\prime}\right)\right\rangle$ can exhibit nonclassical correlations between the atoms that are excluded in our stochastic treatment. Nonclassical saturation phenomena in analogous correlation functions are familiar from inelastic scattering in the resonance fluorescence of a single atom and, e.g., in the formation of the Mollow triplet [62]. In principle, one might improve on this semiclassical approximation by accounting for nonclassical pair correlations by considering the equations of motion for $\rho_{a d ; b c}^{(j, l)}$ in a manner akin to that described in Sec. III B 1.

Under this factorization approximation, the expectation values of Eqs. (26) and (28) for a given set of fixed 
positions $\mathbf{X}_{1}, \ldots, \mathbf{X}_{N}$ of point scatterers then lead to

$$
\begin{aligned}
& \frac{d}{d t} \rho_{g e}^{(j)}=(i \bar{\Delta}-\gamma) \rho_{g e}^{(j)}-i \frac{\xi}{\mathcal{D}}\left(2 \rho_{e e}^{(j)}-1\right) \hat{\mathbf{e}}^{*} \cdot \mathbf{D}_{F}^{+}\left(\mathbf{X}_{j}\right) \\
& \quad-i \xi\left(2 \rho_{e e}^{(j)}-1\right) \sum_{l \neq j} \hat{\mathbf{e}}^{*} \cdot \mathrm{G}^{\prime}\left(\mathbf{X}_{j}-\mathbf{X}_{l}\right) \hat{\mathbf{e}} \rho_{g e}^{(l)} \\
& \frac{d}{d t} \rho_{e e}^{(j)}=-2 \gamma \rho_{e e}^{(j)}+\frac{2 \xi}{\mathcal{D}} \operatorname{Im}\left[\hat{\mathbf{e}} \cdot \mathbf{D}_{F}^{-}\left(\mathbf{X}_{j}\right) \rho_{g e}^{(j)}\right] \\
& \quad+2 \xi \operatorname{Im}\left[\sum_{l \neq j} \rho_{g e}^{(j)} \hat{\mathbf{e}} \cdot \mathrm{G}^{*}\left(\mathbf{X}_{j}-\mathbf{X}_{l}\right) \hat{\mathbf{e}}^{*} \rho_{e g}^{(l)}\right]
\end{aligned}
$$

where we have made use of $\rho_{e e}^{(j)}+\rho_{g g}^{(j)}=1$ to eliminate $\rho_{g g}^{(j)}$ from Eqs. (66) and (67). Once again, we have assumed the driving field to be in a coherent state, and so under expectation values the operators $\hat{\mathbf{D}}_{F}^{ \pm}$give rise to multiplicative classical coherent fields $\mathbf{D}_{F}^{ \pm}$. This set of $2 N$ nonlinear equations can then be solved for the optical response of a single realization of point scatterers. As in the low light intensity limit, the equations have an intuitive form. For a pointlike two-level atom at position $\mathbf{X}_{j}$, with ground and excited state amplitudes $\rho_{g g}^{(j)}$ and $\rho_{e e}^{(j)}$, respectively, Eq. (66) gives the optical response to the sum of the driving field $\mathbf{D}_{F}^{+}$and fields scattered from each of the other atoms $\mathcal{D} G^{\prime}\left(\mathbf{X}_{i}-\mathbf{X}_{j}\right) \hat{\mathbf{e}} \rho_{g e}^{(l)}$. The stochastic simulation of the total response of the system proceeds as in Sec. III A. A set of atomic positions $\left\{\mathbf{X}_{1}, \ldots, \mathbf{X}_{N}\right\}$ is chosen randomly from the $N$-body probability distribution function, which we assume to be known. The above closed set of nonlinear equations can then be solved numerically, and ensemble averaging over many such realizations generates quantities $\left\langle\hat{\psi}_{e}^{\dagger}(\mathbf{r}) \hat{\psi}_{e}(\mathbf{r})\right\rangle,\left\langle\hat{\psi}_{g}^{\dagger}(\mathbf{r}) \hat{\psi}_{g}(\mathbf{r})\right\rangle$, and $\left\langle\hat{\mathbf{P}}^{+}(\mathbf{r})\right\rangle$, detailing the cooperative response of the system. In addition to the $N$-body probability distribution function, the nonlinear nature of the system means that the initial values of $\rho_{e e}^{(j)}$ as well as $\rho_{g e}^{(j)}$ are required, although in the common experimental situation of response to a probe pulse the initial state in the absence of a driving field is trivially $\rho_{g e}^{(j)}=\rho_{e e}^{(j)}=0$.

\section{B. Multilevel atoms}

Realistic experimental situations frequently involve several internal atomic levels that participate in optical probing. In the low light intensity case we generalized the two-level system for the case of multilevel atoms (Sec. III B). In the saturated case we can proceed in the same way and generalize the saturated two-level case of the previous section to a multilevel formalism. In contrast to the low intensity case of Sec. III B, at higher intensities the light may drive dynamics between the relative populations of different internal levels, complicating the analysis. Generalizing the earlier decomposition for a single realization of point scatterers, and to account for the dynamics of level populations, we write

$$
\left\langle\hat{\psi}_{a \nu}^{\dagger} \hat{\psi}_{b \eta}\right\rangle_{\left\{\mathbf{X}_{1}, \ldots, \mathbf{X}_{N}\right\}}=\sum_{j} \rho_{a \nu b \eta}^{(j)} \delta\left(\mathbf{r}-\mathbf{X}_{j}\right)
$$

where $a, b=e, g$. Taking expectation values of Eqs. (24) for a given realization of discrete atomic positions now leads to the equations for the response

$$
\begin{aligned}
& \frac{d}{d t} \rho_{g \nu e \eta}^{(j)}=\left(i \bar{\Delta}_{g \nu e \eta}-\gamma\right) \rho_{g \nu e \eta}^{(j)} \\
& \quad+i \frac{\xi}{\mathcal{D}^{2}} \rho_{g \nu g \tau}^{(j)} \mathbf{d}_{e \eta g \tau} \cdot \mathbf{D}_{F}^{+}\left(\mathbf{X}_{j}\right) \\
& \quad-i \frac{\xi}{\mathcal{D}^{2}} \rho_{e \tau e \eta}^{(j)} \mathbf{d}_{e \tau g \nu} \cdot \mathbf{D}_{F}^{+}\left(\mathbf{X}_{j}\right) \\
& \quad+i \frac{\xi}{\mathcal{D}^{2}} \sum_{l \neq j} \mathbf{d}_{e \eta g \tau} \cdot \mathrm{G}^{\prime}\left(\mathbf{X}_{j}-\mathbf{X}_{l}\right) \mathbf{d}_{g \mu e \zeta} \rho_{g \mu e \zeta}^{(l)} \rho_{g \nu g \tau}^{(j)} \\
& \quad-i \frac{\xi}{\mathcal{D}^{2}} \sum_{l \neq j} \mathbf{d}_{e \tau g \nu} \cdot \mathrm{G}^{\prime}\left(\mathbf{X}_{j}-\mathbf{X}_{l}\right) \mathbf{d}_{g \mu e \zeta} \rho_{g \mu e \zeta}^{(l)} \rho_{e \tau e \eta}^{(j)}
\end{aligned}
$$

$$
\begin{aligned}
& \frac{d}{d t} \rho_{g \nu g \eta}^{(j)}=i \bar{\Delta}_{g \nu g \eta} \rho_{g \nu g \eta}^{(j)}+2 \gamma \mathcal{C}_{\eta,(\eta+\sigma)}^{(\sigma)} \mathcal{C}_{\nu,(\nu+\sigma)}^{(\sigma)} \rho_{e(\nu+\sigma) e(\eta+\sigma)}^{(j)} \\
& \quad-i \frac{\xi}{\mathcal{D}^{2}} \rho_{e \tau g \eta}^{(j)} \mathbf{d}_{e \tau g \nu} \cdot \mathbf{D}_{F}^{+}\left(\mathbf{X}_{j}\right) \\
& \quad+i \frac{\xi}{\mathcal{D}^{2}} \rho_{g \nu e \tau}^{(j)} \mathbf{d}_{g \eta e \tau} \cdot \mathbf{D}_{F}^{-}\left(\mathbf{X}_{j}\right) \\
& \quad-i \frac{\xi}{\mathcal{D}^{2}} \sum_{l \neq j} \mathbf{d}_{e \tau g \nu} \cdot \mathbf{G}^{\prime}\left(\mathbf{X}_{j}-\mathbf{X}_{l}\right) \mathbf{d}_{g \mu e \zeta} \rho_{g \mu e \zeta}^{(l)} \rho_{e \tau g \eta}^{(j)} \\
& \quad+i \frac{\xi}{\mathcal{D}^{2}} \sum_{l \neq j} \mathbf{d}_{g \eta e \tau} \cdot \mathrm{G}^{\prime *}\left(\mathbf{X}_{j}-\mathbf{X}_{l}\right) \mathbf{d}_{e \zeta g \mu} \rho_{e \zeta g \mu}^{(l)} \rho_{g \nu e \tau}^{(j)}
\end{aligned}
$$

$$
\begin{aligned}
& \frac{d}{d t} \rho_{e \nu e \eta}^{(j)}=\left(i \bar{\Delta}_{e \nu e \eta}-2 \gamma\right) \rho_{e \nu e \eta}^{(j)} \\
& \quad+i \frac{\xi}{\mathcal{D}^{2}} \rho_{e \nu g \tau}^{(j)} \mathbf{d}_{e \eta g \tau} \cdot \mathbf{D}_{F}^{+}\left(\mathbf{X}_{j}\right) \\
& \quad-i \frac{\xi}{\mathcal{D}^{2}} \rho_{g \tau e \eta}^{(j)} \mathbf{d}_{g \tau e \nu} \cdot \mathbf{D}_{F}^{-}\left(\mathbf{X}_{j}\right) \\
& \quad-i \frac{\xi}{\mathcal{D}^{2}} \sum_{l \neq j} \mathbf{d}_{g \tau e \nu} \cdot \mathrm{G}^{\prime *}\left(\mathbf{X}_{j}-\mathbf{X}_{l}\right) \mathbf{d}_{e \zeta g \mu} \rho_{e \zeta g \mu}^{(l)} \rho_{g \tau e \eta}^{(j)} \\
& \quad+i \frac{\xi}{\mathcal{D}^{2}} \sum_{l \neq j} \mathbf{d}_{e \eta g \tau} \cdot \mathrm{G}^{\prime}\left(\mathbf{X}_{j}-\mathbf{X}_{l}\right) \mathbf{d}_{g \mu e \zeta} \rho_{g \mu e \zeta}^{(l)} \rho_{e \nu g \tau}^{(j)}
\end{aligned}
$$

where the repeated indices $\tau, \zeta, \mu, \sigma$ are implicitly summed over, and $\bar{\Delta}_{a \nu b \eta}=\Delta_{b \eta}-\Delta_{a \nu}$. In the case of a conserved total atom population, one of the equations can be eliminated by the relation $\sum_{\nu} \rho_{g \nu g \nu}^{(j)}+\sum_{\eta} \rho_{\text {enen }}^{(j)}=$ 1. As in the two-level case, we have introduced a semiclassical approximation to factorize internal level twobody correlation functions in the manner of Eq. (65). 
Due to this approximation, as discussed in the two-level case of the previous section, the ensemble average of many single realizations does not reproduce the nonclassical correlations in the system. To facilitate comparison with the low light intensity results of Sec. III B, we note that the induced electric dipole of atom $j$ for the optical transition $|g, \nu\rangle \rightarrow|e, \eta\rangle$ is $\mathbf{d}_{g \nu e \eta} \rho_{g \nu e \eta}^{(j)}$, which corresponds to the quantity $\mathcal{D} \hat{\mathbf{e}}_{\eta-\nu} \mathcal{C}_{\nu, \eta}^{(\eta-\nu)} f_{\nu}^{(j)} \mathcal{P}_{\nu \eta}^{(j)}$ in the notation of Sec. III B.

The result is a set of coupled equations (69) for internal level one-body density matrix elements $\rho_{a \nu b \eta}^{(j)}$, for each atom $j=1, \ldots, N$. We stochastically sample the set of discrete atomic coordinates $\left\{\mathbf{X}_{1}, \ldots, \mathbf{X}_{N}\right\}$ from the $N$ body density distribution function. For each realization we then solve the semiclassical electrodynamics equations of motion (69) for $\rho_{a \nu b \eta}^{(j)}$. Averaging over many such realizations allows the expectation values of desired observables to be computed. The nonlinear nature of the equations means that the initial values of $\rho_{a \nu b \eta}^{(j)}$ are required that may, e.g., include nonvanishing initial coherences.

\section{NUMERICAL EXAMPLE - ATOMS IN A PERIODIC 2D LATTICE}

In this section, we provide an example of the stochastic simulation techniques described in this paper by studying a Mott-insulator state of atoms confined in a 2D optical lattice that is subjected to incident low-light-intensity excitation. This system has previously been considered in Ref. [17]. We analyze here in detail the phenomena that are most closely related to the present work. We also extend the results of Ref. [17] to calculate the collective excitation eigenmode distributions of the atoms.

We show that the atoms even in a 2D lattice geometry can respond cooperatively when the scattered light mediates strong dipole-dipole interactions between atoms. We find that the optical excitations of the atoms to resonant light are collective and cannot be described by single atom excitation properties. The optical response is then governed by the distinct responses of the various collective modes to the incident field rather than simply the sum of independent atoms. The most subradiant of the collective excitation eigenmodes exhibits radiative resonance linewidths that are dramatically narrowed. Furthermore, the narrowing exhibits a strong dependence on the light-induced interaction strength between the atoms and is more sensitive to the spatial separation of the lattice sites than to the lattice site confinement. The optical response example for a phase-modulated incident field demonstrates how light-induced correlation effects can be employed for engineering optical excitations on a subwavelength scale [17]. These correspond to electromagnetic energy hot-spots whose widths beat the diffraction limit in an analogy to similar examples in solid-state circuit resonator systems [63].

\section{A. Mott-insulator state of atoms in a lattice}

One of the central experimental developments in characterizing strongly interacting phases of ultracold atoms in optical lattices is the ability to manipulate and image atomic spins in individual lattice sites by lasers [64-66]. In this section, we use the classical electrodynamics simulations of point dipoles of Sec. III A to study the optical response of a Mott-insulator state of atoms confined in a 2D optical lattice. For simplicity, we consider two-level atoms when there is precisely one atom per lattice site. Single-atom site-occupancy can be realized, for example, by cooling bosonic atoms to the typical 'wedding-cake' Mott-insulator ground state of an optical lattice superposed on a weak harmonic trap, and manipulating the sites with excess occupancy [66].

We consider an $N_{x} \times N_{y} 2 \mathrm{D}$ square optical lattice with lattice spacing $a$ and sites labeled by index $i=$ $1, \ldots, N\left(N=N_{x} N_{y}\right)$, centered on positions $\mathbf{R}_{i}$ in the $x y$ plane. Each site has a potential depth $s E_{R}$, where $E_{R}=\pi^{2} \hbar^{2} /\left(2 m a^{2}\right)$ is the lattice-photon recoil energy [67]. The lattice sites are sufficiently deep that the optical lattice potential around $\mathbf{R}_{i}$ is well approximated by a harmonic oscillator with vibrational frequency $\omega=$ $2 \sqrt{s} E_{R} / \hbar$. Each occupied site $i$ contains a single atom in the vibrational ground state which is described by the Wannier function $\phi_{i}(\mathbf{r}) \equiv \phi\left(\mathbf{r}-\mathbf{R}_{i}\right)$, where $\phi(\mathbf{r})$ is approximately the ground state of the harmonic oscillator potential

$$
\phi(\mathbf{r}) \simeq \frac{1}{\left(\pi^{3} \ell_{x}^{2} \ell_{y}^{2} \ell_{z}^{2}\right)^{1 / 4}} \exp \left(-\frac{x^{2}}{2 \ell_{x}^{2}}-\frac{y^{2}}{2 \ell_{y}^{2}}-\frac{z^{2}}{2 \ell_{z}^{2}}\right)
$$

The widths of the wavefunction in the lattice are $\ell_{x}=$ $\ell_{y}=\ell=a s^{-1 / 4} / \pi$, and we have assumed an additional oblate external potential confines the atoms in a region around the $z=0$ plane with thickness $\ell_{z}$. For simplicity in the following examples, we assume $\ell_{z} \ll \ell$, and neglect fluctuations of atomic position in the $z$ direction. The atom density, $\rho_{i}(\mathbf{r}) \equiv\left|\phi_{i}(\mathbf{r})\right|^{2}$, at site $i$ thus has a Gaussian profile with a $1 / e$ radius $\ell$ in the $x y$ plane. This radius is directly proportional to the lattice spacing and narrows with increased trapping strength $s$.

\section{B. Light-atom interactions}

In the low light intensity limit, the atoms behave as harmonic oscillators, as described in Sec. III. Here we assume the atoms in each site have a two-level structure with ground state $|g\rangle$, excited state $|e\rangle$, and all other levels shifted out of resonance as described in Sec. III A. The orientation $\hat{\mathbf{e}} \approx \hat{\mathbf{e}}_{z}+0.1 \hat{\mathbf{e}}_{y}$ of the atomic dipole transition is rotated slightly from the normal to the lattice, and the dipole amplitude of atom $j$ is governed by the oscillator variable $\mathcal{P}^{(j)}$. The fields emitted from each atom drive oscillations in the others, and mediate interactions that produce the cooperative optical response. The dynamics 
are governed by Eq. (42), which can be expressed in the form

$$
\frac{d}{d t} \mathcal{P}^{(j)}=\sum_{l=1}^{N} \mathcal{M}_{j, l} \mathcal{P}^{(l)}+F^{(j)},
$$

where the matrix $\mathcal{M}$ accounts for the single atom decay, atomic level shifts, and the light-mediated interactions between different atoms. The driving caused by the incident field is represented by $F^{(j)}$. Each eigenvector of $\mathcal{M}$ corresponds to a collective mode whose collective shift $\delta_{c}$ and decay rate $\Gamma$ are determined by the mode's eigenvalue; $\Upsilon=-\Gamma-i \delta_{c}[16,17]$.

\section{Sampling of atomic positions}

The stochastic realizations of at $\left\{\mathbf{X}_{1}, \mathbf{X}_{2}, \ldots, \mathbf{X}_{N}\right\}$ are sampled from the distribution determined by the many-k 8 tion $P\left(\mathbf{r}_{1}, \ldots, \mathbf{r}_{N}\right)=\left|\Psi\left(\mathbf{r}_{1}, \ldots, \mathbf{r}_{N}\right)\right|^{2}$ We consider the limit where the mor distinct lattice sites $i_{1}$ and $i_{2}$ have ne $\phi_{i_{1}}(\mathbf{r}) \phi_{i_{2}}(\mathbf{r}) \simeq 0$ for all $\mathbf{r}$. In this $\lim$ square of the symmetrized many-body bc tion is

$$
\left|\Psi\left(\mathbf{r}_{1}, \ldots, \mathbf{r}_{N}\right)\right|^{2} \simeq \frac{1}{N !} \sum_{i_{1} \ldots i_{N}}\left|\phi_{i_{1}}\left(\mathbf{r}_{1}\right)\right|^{2}
$$

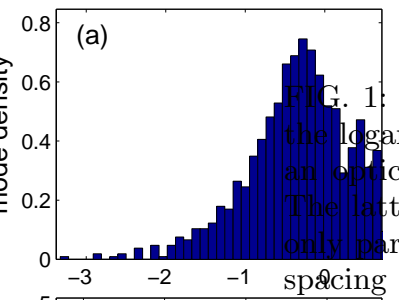

(b)

The probability dis bution (normalized to one) of arithm of collective hy rates for two-level atoms in callattice with dip prientations $\hat{\mathbf{e}} \approx \hat{\mathbf{e}}_{z}+0.1 \hat{\mathbf{e}}_{y}$. tice spacing is $a=(a, b, d)$, while the lattice is rtially populated c with an effective lattice

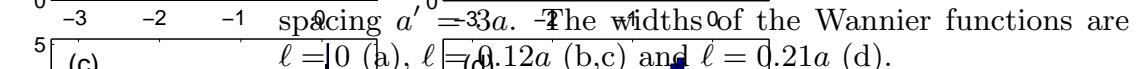

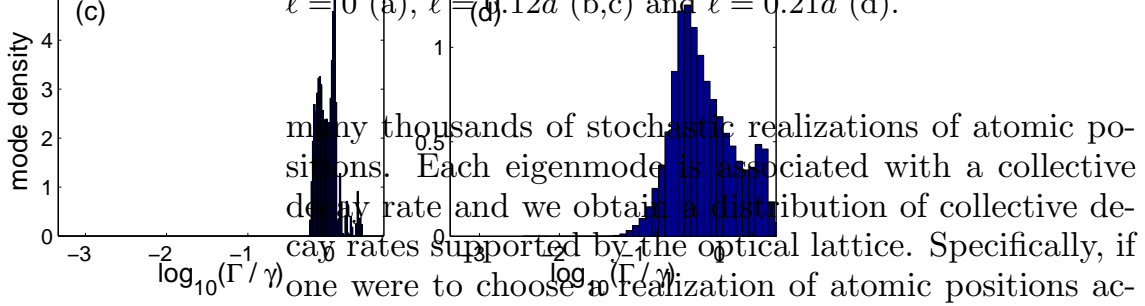
where the summation runs over $N$-tuples responding to all permutations of sites $i=1, \ldots, 1 \mathrm{v}$ coultaining an atom. Rather than sampling the positions of atoms $\mathbf{r}_{j}$ labeled by index $j$ (which could be in any occupied site $i$ ), one can equivalently sample the positions $\overline{\mathbf{r}}_{i}$ of the atom in occupied site $i$ (which could correspond to an atom labeled by any index $j$ ) from the joint probability distribution $\bar{P}\left(\overline{\mathbf{r}}_{1}, \ldots, \overline{\mathbf{r}}_{N}\right)$. Since the many-body correlation function $P$ is invariant under permutation of atomic positions, the joint probability distribution for atomic positions within particular lattice sites is

$$
\begin{aligned}
\bar{P}\left(\overline{\mathbf{r}}_{1}, \ldots, \overline{\mathbf{r}}_{N}\right) & =\sum_{i_{1}, \ldots, i_{N}} P\left(\overline{\mathbf{r}}_{i_{1}}, \ldots, \overline{\mathbf{r}}_{i_{N}}\right) \\
& =\left|\phi_{1}\left(\overline{\mathbf{r}}_{1}\right)\right|^{2} \ldots\left|\phi_{N}\left(\overline{\mathbf{r}}_{N}\right)\right|^{2} .
\end{aligned}
$$

Here, again, the $N$-tuples $\left(i_{1}, \ldots, i_{N}\right)$ are summed over all permutations of sites $i=1, \ldots, N$, and we have taken advantage of the fact that, since atoms are well-localized within their respective sites, $\left|\phi_{i_{1}}\left(\overline{\mathbf{r}}_{i_{2}}\right)\right|^{2}$ is only nonzero when $i_{1}=i_{2}$. For each stochastic realization, we can thus treat the positions of atoms within each site as independent Gaussian random variables with mean $\mathbf{R}_{i}$ and variance $\ell^{2} / 2$ in the $x y$ plane.

\section{Collective excitation eigenmodes}

To characterize the cooperative properties of the system, we calculate its collective excitation eigenmodes for

cording to Eq. (73) and, given those positions, randomly select a collective mode of excitation with collective decay rate $\Gamma$, the probability density of obtaining a particular value of $\log _{10}(\Gamma / \gamma)$ corresponds to the densities shown in Fig. 1. Specifically, Figs. 1(a,b,d) show the distribution for a $32 \times 32$ optical lattice with lattice spacing $a=0.55 \lambda$. When the lattice is infinitely deep (Fig. 1a), the atoms are perfectly confined at the bottom of the lattice sites, and collective decay rates range from the very subradiant $4.7 \times 10^{-4} \gamma$ to the superradiant $6.5 \gamma$. The fluctuations in atomic positions when $\ell \simeq 0.12 a$ (Fig. 1b), associated with lattice depth of $s=50$, narrow the distribution of collective decay rates, which range between $0.017 \gamma$ and $6.3 \gamma$. The relatively shallow lattice with $s=5$ $(\ell \simeq 0.21 a)$ (Fig. 1d) largely preserves the distribution of decay rates calculated for a depth $s=50$. Weakening the confinement strength from $s=50$ to $s=5$ reduces the density of subradiant modes with decay rates below about $0.1 \gamma$. However, the weakly confined lattice still permits strongly subradiant modes. For the realizations of atomic positions we sampled in the shallow $(s=5)$ lattice, decay rates range from $3.5 \times 10^{-3} \gamma$ to $5.9 \gamma$. The distributions spanning several orders of magnitude indicate the atoms in the lattice exhibit strong light-induced correlations.

On the other hand, reducing the density of the atoms has a more deleterious effect on the width of the distribution of collective decay rates than reducing the tight- 
ness of confinement. Figure 1c shows the distribution of collective decay rates in a lattice in which only every third lattice site along the $x$ or $y$ directions is occupied. In effect, the lattice spacing is widened to $a^{\prime}=3 a=1.65 \lambda$. Where the lattice with subwavelength spacing exhibited subradiant collective decay rates suppressed by more than 3 orders of magnitude, the decay rates in the sparsely populated lattice range from $0.55 \gamma$ to $2.6 \gamma$. When the atom density drops, light-induced correlations are weakened.

\section{E. Response to incident light}

Next we compute the response of the lattice to a phasemodulated incident field [17]. Specifically, we consider an $18 \times 18$ lattice $(a=0.55 \lambda)$ with $s=50$. For each realization of atomic positions, we compute the atomic dipole amplitudes $\mathcal{P}^{(i)}$ in each site $i$ from the steadystate solution of Eq. (71). The external field drives each atom with equal amplitude but with a phase $\varphi$ that varies with the $x$ and $y$ coordinates of the atomic positions as

$$
\varphi(x, y) \approx \frac{\pi}{2} \sin \left(2 \pi \frac{x}{\Lambda a}\right) \sin \left(2 \pi \frac{y}{\Lambda a}\right) .
$$

The incident field is only approximately represented by a field with constant amplitude and sinusoidally modulated phase because such a phase-modulation would involve evanescent waves - a more accurate representation may be obtained by a truncated superposition of plane waves as discussed in Ref. [17]. Figure 2a shows the response of a system, in which each lattice site contains one atom, to such a field tuned to the single-atom resonance. The modulation has a period of six lattice sites $(\Lambda=6)$. As a consequence of cooperative interactions, the phase modulated driving excites a superposition of collective modes generating a checkerboard pattern of the average excitation intensities $\left\langle\left|\mathcal{P}^{(i)}\right|^{2}\right\rangle$ in which an atom at every sixth lattice site along each direction is strongly excited. The widths (full width at half maximum) of the excitations are less than the wavelength $0.9 \lambda$. When the effective lattice spacing is tripled to $a^{\prime}=3 a$ as discussed above, however, the light-induced correlations are weakened. A modulation with a comparable period of $6 a^{\prime}(\Lambda=18)$ results in uniform excitation of sites inside the lattice, with atoms at the edge being strongly excited due to boundary effects, as shown in Fig. 2. The lattice spacing that produces a wide distribution of collective decay rates (as in Fig. 1b) results in strong light-induced correlations between the atoms in Fig. 2a, while the lattice with a narrow distribution (as in Fig. 1b) does not show similar behavior.

\section{CONCLUDING REMARKS}

We have derived stochastic simulation techniques for the electrodynamics of atomic ensembles that scale favorably with atom number and are suitable also for dense
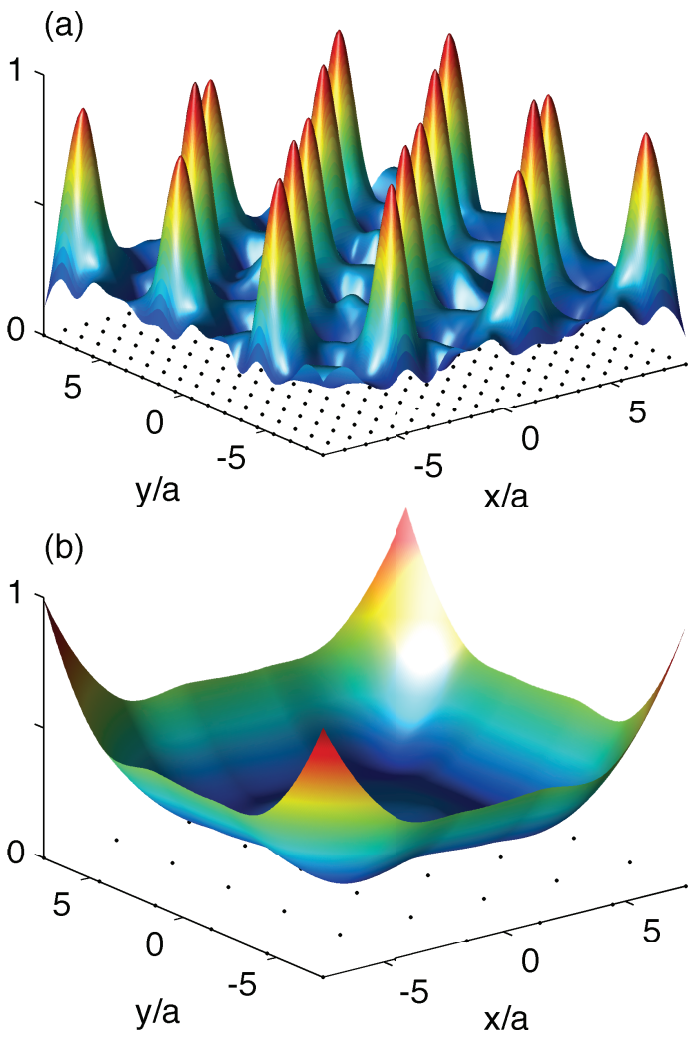

FIG. 2: The atomic dipole intensities $\left\langle\left|\mathcal{P}^{(i)}\right|^{2}\right\rangle / \max _{i^{\prime}}\left\langle\left|\mathcal{P}^{\left(i^{\prime}\right)}\right|^{2}\right\rangle$ in lattice sites $i$. Panel (a) shows strong light-induced correlations between the atoms in a lattice with spacing $a=0.55 \lambda$ and depth $s=50$ to a phase-modulated driving [Eq. (74)] with the modulation period $6 a$. Panel (b) illustrates the absence of cooperative response to a driving with modulation period $6 a^{\prime}$ when only every third lattice site along each direction is occupied, giving an effective spacing of $a^{\prime}=3 a$.

atomic gases and for light that is close to the atomic resonance frequency. We first formulated quantum fieldtheoretical equations of motion describing the optical response [Eqs. (24)] that systematically include the atomic saturation effects and the internal level structure. In order to solve the resultant dynamics, we then introduced a simple procedure to derive stochastic electrodynamics equations that can be used to study recurrent, or dependent, scattering between the atoms in the presence of strong light-atom coupling. In the low light intensity limit, stochastically sampling the positions of atoms from the appropriate $N$-body density distribution function leads to a model of classical coupled linear harmonic oscillators. The solution of this model, and subsequent averaging over a large number of realizations, generates results for the optical response of the atomic ensemble. The solution for coherently scattered light is exact for stationary atoms possessing only a single electronic ground level, since the averaged results reproduce the correct dynamics for the many-body polarization density correlation functions to all orders, as shown in App. B. We have extended this technique to deal with atoms with 
multiple ground levels, and situations where saturation effects are important.

In deriving our stochastic classical electrodynamics simulations, we have first removed the spatial dependence from many-body correlation functions by considering hypothetical cases of fixed atomic positions, sampling those positions from the $N$-body joint probability distribution. Averaging over many realizations later restores the spatial aspect of the correlations, incorporating recurrent scattering processes in the atomic response. For a single realization, the remaining many-body correlations are between internal levels. To obtain numerically efficient simulations we have factorized such internal-level correlations. In general, this factorization can neglect some many-body internal-level quantum correlations, and is therefore only approximately valid. The exception to this is the case of atoms with a single ground level in the limit of low light intensity, where the stochastic simulations give the exact response for the coherent scattering of light from an ensemble of stationary atoms. Physically, this stems from the fact that there are no dynamics of the excited populations (due to low intensity) and there are no other ground levels involved in the optical transitions that could become correlated. Therefore, for such a system the excitation of each atom can be described by a linear polarisation amplitude, with no additional internal-level dynamics possible. The dynamics is equal to that of a coupled system of charged classical linear harmonic oscillators and light, the system modeled by the stochastic electrodynamics simulations in this limit [Eq. (36)].

In contrast, for atoms with multiple electronic ground levels, and/or in the presence of saturation, the factorization of pair correlations provides a technique that remains efficient and scales favorably with atom number, but at a cost of neglecting some quantum internal-level many-body correlations. We emphasize that in such situations, while some internal-level pair correlations are not exactly reproduced, the ensemble averaging over many stochastic realizations still incorporates recurrent scattering into the results of the stochastic simulations (and, for example, for the case of the low light intensity limit multilevel system, the classical simulation approximation correctly predicts the expectation values of the different ground-state populations). We have additionally shown how one can improve on the approximate methods obtained by this factorization by considering the dynamics of the internal-level pair correlations, at a cost of a more complicated numerical system of equations.

Light-induced correlation phenomena in wave propagation are strongly enhanced in cold homogeneously broadened samples. Consequently, the stochastic simulations techniques that incorporate recurrent scattering events between closely-spaced atoms are most suitable in the systems where the inhomogeneous broadening (e.g., due to Doppler shifts) does not significantly exceed the radiative linewidth of the atoms. In this limit the inhomogeneous broadening has been shown to suppress the light- induced correlations [21], resulting in an optical response that is more closely reminiscent of that of a standard continuous medium electrodynamics.

The stochastic simulation methods replace the difficult problem of deriving, and solving, many coupled equations of motion for up to $N$-body correlations with a stochastic approach that relies on sampling atomic positions. If the atoms are initially uncorrelated before the light enters the medium, the position of each atom becomes an independent random variable that is sampled from the atom density distribution. In the presence of nontrivial position correlations for the atoms the sampling from the correct many-body density operator for the system can become challenging. An ensemble of stochastic atomic positions must be synthesized which generates the corresponding physical position correlations between the atoms. While we have here investigated a relatively simple case of a Mott-insulator state in an optical lattice (Sec. V), systems with more complicated density correlation functions, for example Fermi-Dirac statistics, can also be sampled by Monte-Carlo simulation methods [10].

\section{Acknowledgments}

This research was supported by the EPSRC and the Leverhulme trust.

\section{APPENDIX A: EVALUATION OF THE ATOM-LIGHT COMMUTATORS}

Here we demonstrate the calculation of the commutators between the atomic field operators and the free electric displacement. Consider $\left[\hat{\mathbf{D}}_{F}^{+}(\mathbf{r}), \hat{\psi}_{g \nu}\left(\mathbf{r}^{\prime}\right)\right]$. The total electric displacement $\hat{\mathbf{D}}^{+}(\mathbf{r})=\hat{\mathbf{D}}_{F}^{+}(\mathbf{r})+\hat{\mathbf{D}}_{S}^{+}(\mathbf{r})$ is a canonical momentum of the light field and commutes with the atomic field operators. Hence

$$
\begin{aligned}
{\left[\hat{\mathbf{D}}_{F}^{+}(\mathbf{r}), \hat{\psi}_{g \nu}\left(\mathbf{r}^{\prime}\right)\right]=} & -\left[\hat{\mathbf{D}}_{S}^{+}(\mathbf{r}), \hat{\psi}_{g \nu}\left(\mathbf{r}^{\prime}\right)\right] \\
= & -\int d^{3} r^{\prime} \mathrm{G}\left(\mathbf{r}-\mathbf{r}^{\prime \prime}\right)\left[\hat{\mathbf{P}}^{+}\left(\mathbf{r}^{\prime \prime}\right), \hat{\psi}_{g \nu}\left(\mathbf{r}^{\prime}\right)\right] \\
& -\left[\hat{\mathbf{P}}^{+}(\mathbf{r}), \hat{\psi}_{g \nu}\left(\mathbf{r}^{\prime}\right)\right] .
\end{aligned}
$$

Applying then the standard commutation relations, such as

$$
\left[\hat{\psi}_{g \tau}(\mathbf{r}), \hat{\psi}_{g \nu}^{\dagger}\left(\mathbf{r}^{\prime}\right)\right]=\delta\left(\mathbf{r}-\mathbf{r}^{\prime}\right) \delta_{\tau, \nu}
$$

yields the result of Eq. (18).

\section{APPENDIX B: MONTE-CARLO EVALUATION OF CORRELATION FUNCTIONS IN THE LOW LIGHT INTENSITY LIMIT}

By treating atoms and light as quantum fields throughout the analysis, it was shown in Ref. [7] that a coop- 
erative coupling between atoms and light leads to coupled equations of motion between correlation functions of atomic density and polarization. In the limit of low light intensity the resulting hierarchy of equations represents strong light-mediated correlations that become important at low temperatures and in dense sampleson the other hand, for inhomogeneously broadened hot atoms the standard mean-field theory of continuous effective medium electrodynamics can be restored [21]. It was shown in Ref. [10] for a simplified 1D scalar electrodynamics how stochastically sampling atomic positions, calculating the collective response for atoms at fixed positions, and averaging over those stochastic realizations, reproduces the dynamics described by these correlation functions. In this Appendix, we extend the quantum field-theoretical representation that is expressed in terms of the hierarchy of correlation functions [7] to the general case of atoms with multiple electronic ground levels. We then briefly generalize the treatment of Ref. [10] to the $3 \mathrm{D}$ electrodynamics including the full vector properties of the electromagnetic fields for the special case of atoms with the $J=0 \rightarrow J^{\prime}=1$ transition, corresponding to an isotropic susceptibility. The analysis is similar to other atomic level systems that have only a single electronic ground state, and shows how the coupled dynamics of correlation functions in the presence of a single electronic ground level is reproduced by the classical electrodynamics simulations.

We begin by considering the hierarchy of correlation functions. In the low light-intensity limit, the equation of motion for polarization simplifies to [Eq. (29)]

$$
\begin{aligned}
\frac{d}{d t} \hat{\mathbf{P}}_{\nu \eta}^{+} & =\left(i \bar{\Delta}_{g \nu e \eta}-\gamma\right) \hat{\mathbf{P}}_{\nu \eta}^{+}+i \xi \hat{\psi}_{g \nu}^{\dagger} \hat{\psi}_{g \tau} \mathbf{P}_{\eta \tau}^{\nu \eta} \hat{\mathbf{D}}_{F}^{+} \\
& +i \xi \int d^{3} r^{\prime} \mathbf{P}_{\eta \tau}^{\nu \eta} \mathrm{G}^{\prime}\left(\mathbf{r}-\mathbf{r}^{\prime}\right) \hat{\psi}_{g \nu}^{\dagger} \hat{\mathbf{P}}^{+}\left(\mathbf{r}^{\prime}\right) \hat{\psi}_{g \tau}
\end{aligned}
$$

To determine the average scattered fields in the low light intensity limit, it is necessary to find the average polarization within the atomic ensemble. Taking the expectation value of Eq. (B1), however, one finds that the dynamics of the polarization depend, not simply on the polarization density in the ensemble, but on a two-body correlation function $\left\langle\hat{\psi}_{g \nu}^{\dagger}(\mathbf{r}) \hat{\mathbf{P}}^{+}\left(\mathbf{r}^{\prime}\right) \hat{\psi}_{g \tau}(\mathbf{r})\right\rangle$. It was shown in Ref. [7] that in the low light intensity limit, in order to solve the second-order correlation function, one has to further find the dynamics of a third-order correlation function; a third-order correlation function requires a fourth-order correlation function, and so on. The result is a hierarchy of dynamic equations of motion for the $\ell^{\text {th }}$ order correlation functions

$$
\begin{aligned}
\rho_{\ell}^{\nu_{1} \tau_{1}, \ldots, \nu_{\ell} \tau_{\ell}}\left(\mathbf{r}_{1}, \ldots, \mathbf{r}_{\ell}\right) & \equiv\left\langle\hat{\psi}_{g \nu_{1}}^{\dagger}\left(\mathbf{r}_{1}\right) \ldots \hat{\psi}_{g \nu_{\ell}}^{\dagger}\left(\mathbf{r}_{\ell}\right) \hat{\psi}_{g \tau_{\ell}}\left(\mathbf{r}_{\ell}\right) \ldots \hat{\psi}_{g \tau_{1}}\left(\mathbf{r}_{1}\right)\right\rangle \\
P_{\ell}^{\nu_{1} \tau_{1}, \ldots, \nu_{\ell-1} \tau_{\ell-1} ; \nu_{\ell} \eta_{\ell}}\left(\mathbf{r}_{1}, \ldots, \mathbf{r}_{\ell-1} ; \mathbf{r}_{\ell}\right) & \equiv\left\langle\hat{\psi}_{g \nu_{1}}^{\dagger}\left(\mathbf{r}_{1}\right) \ldots \hat{\psi}_{g \nu_{\ell-1}}^{\dagger}\left(\mathbf{r}_{\ell-1}\right) \hat{\psi}_{g \nu_{\ell}}^{\dagger}\left(\mathbf{r}_{\ell}\right) \hat{\psi}_{e \eta_{\ell}}\left(\mathbf{r}_{\ell}\right) \hat{\psi}_{g \tau_{\ell-1}}\left(\mathbf{r}_{\ell-1}\right) \ldots \hat{\psi}_{g \tau_{1}}\left(\mathbf{r}_{1}\right)\right\rangle .
\end{aligned}
$$

The quantum-mechanical ground-level many-body correlation function $\rho_{\ell}^{\nu_{1} \tau_{1}, \ldots, \nu_{\ell} \tau_{\ell}}\left(\mathbf{r}_{1}, \ldots, \mathbf{r}_{\ell}\right)$ represents the spatial correlations in the absence of incident light. These correlation functions are constants of motion in the low light intensity limit, and zero magnetic field. The presence of a constant magnetic field induces a phase-rotation owing to Zeeman splitting. Specifically,

$$
\frac{d}{d t} \rho_{\ell}^{\nu_{1} \tau_{1}, \ldots, \nu_{\ell} \tau_{\ell}}\left(\mathbf{r}_{1}, \ldots, \mathbf{r}_{\ell}\right)=i\left[\sum_{k=1}^{\ell} \bar{\Delta}_{g \nu_{k} g \tau_{k}}\right] \rho_{\ell}^{\nu_{1} \tau_{1}, \ldots, \nu_{\ell} \tau_{\ell}}\left(\mathbf{r}_{1}, \ldots, \mathbf{r}_{\ell}\right)
$$

To calculate the optical response of the system to the lowest order in the electric field amplitude, we follow the evolution of the $\ell$-atom correlation function $P_{\ell}^{\nu_{1} \tau_{1}, \ldots, \nu_{\ell-1} \tau_{\ell-1} ; \nu_{\ell} \eta_{\ell}}\left(\mathbf{r}_{1}, \ldots, \mathbf{r}_{\ell-1} ; \mathbf{r}_{\ell}\right)$ for the ground-level atoms and/or ground-level coherences at positions $\mathbf{r}_{1}, \ldots, \mathbf{r}_{\ell-1}$, given there is a polarization at position $\mathbf{r}_{\ell}$. These correlation functions satisfy the equations of motion $(\ell=1, \ldots, N)$

$$
\begin{aligned}
& \left\{\frac{d}{d t}-i\left[\bar{\Delta}_{g \nu_{\ell} e \eta_{\ell}}+\sum_{k=1}^{\ell-1} \bar{\Delta}_{g \nu_{k} g \tau_{k}}\right]+\gamma\right\} P_{\ell}^{\nu_{1} \tau_{1}, \ldots, \nu_{\ell-1} \tau_{\ell-1} ; \nu_{\ell} \eta_{\ell}}\left(\mathbf{r}_{1}, \ldots, \mathbf{r}_{\ell-1} ; \mathbf{r}_{\ell}\right)= \\
& i \frac{\xi}{\mathcal{D}} \rho_{\ell}^{\nu_{1} \tau_{1}, \ldots, \nu_{\ell-1} \tau_{\ell-1}, \nu_{\ell} \tau}\left(\mathbf{r}_{1}, \ldots, \mathbf{r}_{\ell}\right) \mathcal{C}_{\tau, \eta_{\ell}}^{(\sigma)} \hat{\mathbf{e}}_{\sigma}^{*} \cdot \mathbf{D}_{F}^{+}\left(\mathbf{r}_{\ell}\right) \\
& +i \xi \sum_{k=1}^{\ell-1} \mathcal{C}_{\tau, \eta_{\ell}}^{(\sigma)} \hat{\mathbf{e}}_{\sigma}^{*} \cdot \mathrm{G}^{\prime}\left(\mathbf{r}_{\ell}-\mathbf{r}_{k}\right) \hat{\mathbf{e}}_{\varsigma} \mathcal{C}_{\tau_{k}, \eta}^{(\varsigma)} \\
& \quad \times P_{\ell}^{\nu_{1} \tau_{1}, \ldots, \nu_{k-1} \tau_{k-1}, \nu_{\ell} \tau, \nu_{k+1} \tau_{k+1}, \ldots, \nu_{\ell-1} \tau_{\ell-1} ; \nu_{k} \eta}\left(\mathbf{r}_{1}, \ldots, \mathbf{r}_{k-1}, \mathbf{r}_{\ell}, \mathbf{r}_{k+1}, \ldots, \mathbf{r}_{\ell-1} ; \mathbf{r}_{k}\right) \\
& +i \xi \int d^{3} r_{\ell+1} \mathcal{C}_{\tau, \eta_{\ell}}^{(\sigma)} \hat{\mathbf{e}}_{\sigma}^{*} \cdot \mathrm{G}^{\prime}\left(\mathbf{r}_{\ell}-\mathbf{r}_{\ell+1}\right) \hat{\mathbf{e}}_{\varsigma} \mathcal{C}_{\nu, \eta}^{(\varsigma)} P_{\ell+1}^{\nu_{1} \tau_{1}, \ldots, \nu_{\ell} \tau ; \nu \eta}\left(\mathbf{r}_{1}, \ldots, \mathbf{r}_{\ell} ; \mathbf{r}_{\ell+1}\right),
\end{aligned}
$$


where repeated indices $\nu, \tau, \eta, \sigma$, and $\varsigma$ are summed over.

Our primary goal in this Appendix is to show that the hierarchy of equations (B5) can be solved exactly (within the statistical accuracy) by classical electrodynamics simulations for the case of a single electronic ground level. (Here we specifically consider the $J=0 \rightarrow J^{\prime}=1$ transition.) These classical simulations account for light-mediated interactions and recurrent (or dependent) scattering between the atoms to all orders. As discussed in Sec. III B, similar classical electrodynamics simulations are only approximate for multilevel atoms, since they do not account for quantum fluctuations between internal levels. For atoms with an isotropic susceptibility, we define the correlation functions

$$
\begin{aligned}
\mathbf{P}_{\ell}\left(\mathbf{r}_{1}, \ldots, \mathbf{r}_{\ell-1} ; \mathbf{r}_{\ell}\right) & \equiv \mathcal{D} \hat{\mathbf{e}}_{\sigma} P_{\ell}^{00, \ldots, 00 ; 0 \sigma}\left(\mathbf{r}_{1}, \ldots, \mathbf{r}_{N}\right), \\
\rho_{\ell}\left(\mathbf{r}_{1}, \ldots, \mathbf{r}_{\ell}\right) & \equiv \rho_{\ell}^{00, \ldots, 00}\left(\mathbf{r}_{1}, \ldots, \mathbf{r}_{\ell}\right),
\end{aligned}
$$

where the repeated index $\sigma$ is summed over. In this simplified case, the many-body ground-level correlation functions are constants of motion, and $\mathbf{P}_{\ell}$ satisfies the equations of motion

$$
\begin{aligned}
& \dot{\mathbf{P}}_{\ell}\left(\mathbf{r}_{1}, \ldots, \mathbf{r}_{\ell-1} ; \mathbf{r}_{\ell}\right)=(i \bar{\Delta}-\gamma) \mathbf{P}_{\ell}\left(\mathbf{r}_{1}, \ldots, \mathbf{r}_{\ell-1} ; \mathbf{r}_{\ell}\right)+i \xi \rho_{\ell}\left(\mathbf{r}_{1}, \ldots, \mathbf{r}_{\ell}\right) \mathbf{D}_{F}^{+}\left(\mathbf{r}_{\ell}\right) \\
& \quad+i \xi \sum_{k=1}^{\ell-1} \mathrm{G}^{\prime}\left(\mathbf{r}_{\ell}-\mathbf{r}_{k}\right) \mathbf{P}_{\ell}\left(\mathbf{r}_{1}, \ldots, \mathbf{r}_{k-1}, \mathbf{r}_{\ell}, \mathbf{r}_{k+1}, \ldots, \mathbf{r}_{\ell-1} ; \mathbf{r}_{k}\right)+i \xi \int d^{3} \mathbf{r}_{\ell+1} \mathrm{G}^{\prime}\left(\mathbf{r}_{\ell}-\mathbf{r}_{\ell+1}\right) \mathbf{P}_{\ell+1}\left(\mathbf{r}_{1}, \ldots, \mathbf{r}_{\ell} ; \mathbf{r}_{\ell+1}\right)
\end{aligned}
$$

Here $\mathbf{P}_{\ell}\left(\mathbf{r}_{1}, \ldots, \mathbf{r}_{\ell-1} ; \mathbf{r}_{\ell}\right)$ is a correlation function for the ground-state atomic positions at $\mathbf{r}_{1}, \ldots, \mathbf{r}_{\ell-1}$, given that there is a polarization at $\mathbf{r}_{\ell}$. The corresponding hierarchy for equations of motion governing two-level atoms can be found from Eq. (B8) by making the replacement $\mathbf{P}_{\ell} \rightarrow \hat{\mathbf{e}} P_{\ell}$. In the limit of low light intensity $\rho_{\ell}$ are not affected by the excitations and are constants of the motion. The ground-state correlations are related to the many-body wave function of the atoms by

$$
\rho_{\ell}\left(\mathbf{r}_{1}, \ldots, \mathbf{r}_{\ell}\right)=\frac{N !}{(N-l) !} \int d^{3} \mathbf{r}_{\ell+1} \ldots d^{3} \mathbf{r}_{N}\left|\Psi\left(\mathbf{r}_{1}, \ldots, \mathbf{r}_{N}\right)\right|^{2} .
$$

For a classical (initially uncorrelated ensemble) or, e.g., a Bose-Einstein condensate, we simply have

$$
\rho_{\ell}\left(\mathbf{r}_{1}, \ldots, \mathbf{r}_{\ell}\right)=\mathcal{N} \rho_{1}\left(\mathbf{r}_{1}\right) \ldots \rho_{1}\left(\mathbf{r}_{\ell}\right),
$$

where $\mathcal{N}$ is a normalisation factor.

Following the treatment of Ref. [10], we introduce an ensemble of classical dipoles at fixed positions $\mathbf{X}_{j}$ for $j=$ $1, \ldots, N$. The individual atomic dipole amplitudes $\mathcal{P}^{(j)}$ then interact via the scattered electromagnetic field and evolve according to Eq. (36),

$$
\frac{d}{d t} \mathcal{P}^{(j)}=(i \bar{\Delta}-\gamma) \mathcal{P}^{(j)}+i \frac{\xi}{\mathcal{D}} \mathbf{D}_{F}^{+}\left(\mathbf{X}_{j}\right)+i \xi \sum_{l \neq j} \mathrm{G}^{\prime}\left(\mathbf{X}_{j}-\mathbf{X}_{l}\right) \mathcal{P}^{(l)}
$$

By means of treating the atomic positions $\left\{\mathbf{X}_{1}, \ldots, \mathbf{X}_{N}\right\}$ as random variables that satisfy an appropriately chosen joint probability distribution $P\left(\mathbf{r}_{1}, \ldots, \mathbf{r}_{N}\right)$, we can construct an ensemble-averaged solution that reproduces dynamics equivalent to the hierarchy of equations of motion (B8). In practise, we solve the coupled equations for the light and atomic dipoles for each stochastic realization of a fixed set of positions and ensemble average the quantities of interest over many such realizations.

We take the joint probability distribution of the positions of the atomic dipoles to be the absolute square of the quantum many-body wave function of the ground-state atoms

$$
P\left(\mathbf{r}_{1}, \ldots, \mathbf{r}_{N}\right)=\left|\Psi\left(\mathbf{r}_{1}, \ldots, \mathbf{r}_{N}\right)\right|^{2} .
$$

With this choice, we may now introduce classical ground-state position correlation functions $\tilde{\rho}_{\ell}\left(\mathbf{r}_{1}, \ldots, \mathbf{r}_{\ell}\right)$ for the atoms that coincide with the normally-ordered quantum-mechanical position correlation functions $\rho_{\ell}\left(\mathbf{r}_{1}, \ldots, \mathbf{r}_{\ell}\right)$. Specifically,

$$
\begin{aligned}
& \tilde{\rho}_{\ell}\left(\mathbf{r}_{1}, \ldots, \mathbf{r}_{\ell}\right) \equiv\left\langle\sum_{j_{1}, \ldots, j_{\ell}}{ }^{\prime} \delta\left(\mathbf{r}_{1}-\mathbf{X}_{j_{1}}\right) \ldots \delta\left(\mathbf{r}_{\ell}-\mathbf{X}_{j_{\ell}}\right)\right\rangle \\
& \quad=\sum_{j_{1}, \ldots, j_{\ell}}^{\prime} \int d^{3} \mathbf{X}_{1}, \ldots, d^{3} \mathbf{X}_{N} P\left(\mathbf{X}_{1}, \ldots, \mathbf{X}_{N}\right) \delta\left(\mathbf{r}_{1}-\mathbf{X}_{j_{1}}\right) \ldots \delta\left(\mathbf{r}_{2}-\mathbf{X}_{j_{\ell}}\right) \\
& =\frac{N !}{(N-\ell) !} \int d^{3} \mathbf{r}_{\ell+1}, \ldots, d^{3} \mathbf{r}_{N} P\left(\mathbf{r}_{1}, \ldots, \mathbf{r}_{N}\right)
\end{aligned}
$$


The prime in the summation indicates that those terms in which any of $j_{1}, \ldots, j_{\ell}$ refer to the same atom are excluded. In the last line we used the fact that the primed summations over the $\ell$-tuples $j_{1}, \ldots, j_{\ell}$ include all $N ! /(N-\ell)$ ! permutations of $\ell$ indices from the set of $N$ atomic indices $\{j=1, \ldots, N\}$. We can therefore in principle simulate the quantum-mechanical position correlations $\rho_{\ell}\left(\mathbf{r}_{1}, \ldots, \mathbf{r}_{\ell}\right)$ by stochastic sampling of the positions of classical dipoles in the hierarchy of equations (B8). In order to show that the hierarchy can be solved by integrating Eq. (B11) for each individual stochastic realization and subsequently ensemble-averaging the results, we proceed as follows. We consider an ensemble of atoms at fixed positions $\mathbf{X}_{j}$ for $j=1, \ldots, N$, multiply the terms in Eq. (B11) by products of delta functions, and sum over the atomic positions to obtain

$$
\begin{aligned}
\left(\frac{d}{d t}+\gamma-i \bar{\Delta}\right) & \mathcal{D} \sum_{j_{1}, \ldots, j_{\ell}}^{\prime} \delta\left(\mathbf{r}_{1}-\mathbf{X}_{j_{1}}\right) \ldots \delta\left(\mathbf{r}_{\ell}-\mathbf{X}_{j_{\ell}}\right) \mathcal{P}^{\left(j_{\ell}\right)}=i \xi \sum_{j_{1}, \ldots, j_{\ell}}^{\prime} \delta\left(\mathbf{r}_{1}-\mathbf{X}_{j_{1}}\right) \ldots \delta\left(\mathbf{r}_{\ell}-\mathbf{X}_{j_{\ell}}\right) \mathbf{D}_{F}^{+}\left(\mathbf{r}_{\ell}\right) \\
& +i \xi \sum_{j_{1}, \ldots, j_{\ell}}^{\prime} \sum_{j_{m} \neq j_{\ell}} \delta\left(\mathbf{r}_{1}-\mathbf{X}_{j_{1}}\right) \ldots \delta\left(\mathbf{r}_{\ell}-\mathbf{X}_{j_{\ell}}\right) \mathcal{D} G^{\prime}\left(\mathbf{r}_{\ell}-\mathbf{X}_{j_{m}}\right) \mathcal{P}^{\left(j_{m}\right)} .
\end{aligned}
$$

There are two types of terms contained in the summation over $j_{m}$ in the last line of Eq. (B15). Either $j_{m}$ corresponds to an index of one of the atoms in the $\ell$-tuples $\left(j_{1}, \ldots, j_{\ell}\right)$ that do not reside at $\mathbf{r}_{\ell}$ (i.e., $j_{m} \in\left\{j_{1}, \ldots, j_{\ell-1}\right\}$ ), or it does not. Accounting for each type of term separately and taking the expectation value, we find that the last line of Eq. (B15) becomes

$$
\begin{aligned}
& i \xi\left\langle\sum_{j_{1}, \ldots, j_{\ell}}^{\prime} \sum_{j_{m} \neq j_{\ell}} \delta\left(\mathbf{r}_{1}-\mathbf{X}_{j_{1}}\right) \ldots \delta\left(\mathbf{r}_{\ell}-\mathbf{X}_{j_{\ell}}\right) \mathcal{D} \mathrm{G}^{\prime}\left(\mathbf{r}_{\ell}-\mathbf{X}_{j_{m}}\right) \mathcal{P}^{\left(j_{m}\right)}\right\rangle \\
& \quad=i \xi \sum_{k=1}^{\ell-1} \mathrm{G}^{\prime}\left(\mathbf{r}_{\ell}-\mathbf{r}_{k}\right) \tilde{\mathbf{P}}_{\ell}\left(\mathbf{r}_{1}, \ldots, \mathbf{r}_{k-1}, \mathbf{r}_{\ell}, \mathbf{r}_{k+1}, \ldots, \mathbf{r}_{\ell-1} ; \mathbf{r}_{k}\right) \\
& \quad+i \xi \int d^{3} \mathbf{r}_{\ell+1} \mathrm{G}^{\prime}\left(\mathbf{r}_{\ell}-\mathbf{r}_{\ell+1}\right) \tilde{\mathbf{P}}_{\ell+1}\left(\mathbf{r}_{1}, \ldots, \mathbf{r}_{\ell} ; \mathbf{r}_{\ell+1}\right)
\end{aligned}
$$

where we have defined the classical correlation function in an analogy to Eq. (B6) as

$$
\tilde{\mathbf{P}}_{\ell}\left(\mathbf{r}_{1}, \ldots, \mathbf{r}_{\ell-1} ; \mathbf{r}_{\ell}\right) \equiv\left\langle\mathcal{D} \sum_{j_{1}, \ldots, j_{\ell}}^{\prime} \delta\left(\mathbf{r}_{1}-\mathbf{X}_{j_{1}}\right) \ldots \delta\left(\mathbf{r}_{\ell}-\mathbf{X}_{j_{\ell}}\right) \mathcal{P}^{\left(j_{\ell}\right)}\right\rangle
$$

The second line of Eq. (B16) accounts for the field scattered from atoms at positions $\mathbf{r}_{1}, \ldots, \mathbf{r}_{\ell-1}$ to the atom at $\mathbf{r}_{\ell}$, and the third line accounts for scattering from other atoms $(\ell+1, \ldots, N)$ in the system to the atom at position $\mathbf{r}_{\ell}$. Taking the average of all terms of Eq. (B15), we obtain the same hierarchy of equations for the classical correlation functions $\tilde{\mathbf{P}}_{\ell}$ and $\tilde{\rho}_{\ell}$, as for the quantum-mechanical correlations $\mathbf{P}_{\ell}$ and $\rho_{\ell}$ in Eq. (B8).

The averages involved in the calculations can be evaluated by Monte-Carlo simulations. One takes many realizations of $N$ atomic positions $\mathbf{X}_{1}, \ldots, \mathbf{X}_{N}$ from the system's initial $N$-body density correlation function. Then, for each realization of random variables, we take the atoms to be localized at the sampled positions so that the correlation functions $\rho_{\ell}$ and $\mathbf{P}_{\ell}$ are described by Eq. (B6). Then, one solves the dynamics of $\mathcal{P}^{(j)}$, which depend on the atomic positions. The correlation functions then emerge as their average over many stochastic realizations.

\section{APPENDIX C: CLASSICAL ELECTRODYNAMICS IN THE MULTILEVEL LOW LIGHT INTENSITY CASE}

Our general approach in this paper addresses spatial correlations in the optical response by fixing the atomic positions in each stochastic realization according to some given probability distribution and then ensemble-averaging over many such realizations. In the case of atoms with multiple electronic ground levels, however, each spatial configuration for the multilevel case still includes the internal level dynamics. In Sec. III B we presented a formalism how to incorporate the internal level dynamics in classical electrodynamics simulations.
The analysis of the internal level many-body correlations in Sec. III B 1 provides quantum corrections to the multilevel dynamics in Eq. (42). We will now show that Eq. (42) involves two types of dynamical processes: (i) classical scattering processes (for which $\nu=\tau$ and $\mu=\beta$ ) that are already incorporated in the classical electrodynamics simulation model of Sec. IIIB; and (ii) virtual quantum processes that go beyond the classical coupled dipole model.

Consider the low light intensity classical multilevel 
electrodynamics model of Eq. (47),

$$
\begin{aligned}
& \frac{d}{d t} \mathcal{P}_{\mu \eta}^{(j)}=\left(i \bar{\Delta}_{g \mu e \eta}-\gamma\right) \mathcal{P}_{\mu \eta}^{(j)}+i \frac{\xi}{\mathcal{D}} \mathcal{C}_{\mu, \eta}^{(\sigma)} \hat{\mathbf{e}}_{\sigma}^{*} \cdot \mathbf{D}_{F}^{+}\left(\mathbf{X}_{j}\right) \\
& \quad+i \xi \sum_{l \neq j} \mathcal{C}_{\mu, \eta}^{(\sigma)} \hat{\mathbf{e}}_{\sigma}^{*} \cdot \mathbf{G}^{\prime}\left(\mathbf{X}_{j}-\mathbf{X}_{l}\right) \hat{\mathbf{e}}_{\varsigma} \mathcal{C}_{\epsilon, \zeta}^{(\varsigma)} f_{\epsilon}^{(l)} \mathcal{P}_{\epsilon \zeta}^{(l)}
\end{aligned}
$$

where we have used the factorization of Eq. (50),

$$
\mathcal{P}_{\nu \tau ; \epsilon \zeta}^{(j ; l)} \simeq \delta_{\nu \tau} f_{\nu}^{(j)} f_{\epsilon}^{(l)} \mathcal{P}_{\epsilon \zeta}^{(l)}
$$

The formula $(\mathrm{C} 1)$ provides the classical electrodynamics simulation model for time-dependent multilevel systems. Next we replace $j$ by $j_{2}$ and $l$ by $j_{3}$ in Eq. (C1) and multiply the both sides of Eq. (C1) by $f_{\nu}^{\left(j_{1}\right)} f_{\mu}^{\left(j_{2}\right)}$, indicating that the atom $j_{1}$ is in the level $\nu$ and $j_{2}$ in $\mu$. In the last term we have to deal separately with the terms $j_{1}=j_{3}$ and $j_{1} \neq j_{3}$. Noting that the atom $j_{1}$ occupies $\nu$, we obtain

$$
\begin{aligned}
& {\left[\frac{d}{d t}-i \bar{\Delta}_{g \mu e \eta}+\gamma\right] \mathcal{P}_{\nu \nu ; \mu \eta}^{\left(j_{1} ; j_{2}\right)}=i \frac{\xi}{\mathcal{D}} f_{\nu}^{\left(j_{1}\right)} f_{\mu}^{\left(j_{2}\right)} \mathcal{C}_{\mu, \eta}^{(\sigma)} \hat{\mathbf{e}}_{\sigma}^{*} \cdot \mathbf{D}_{F}^{+}\left(\mathbf{X}_{j_{2}}\right)} \\
& +i \xi \mathcal{C}_{\mu, \eta}^{(\sigma)} \hat{\mathbf{e}}_{\sigma}^{*} \cdot G^{\prime}\left(\mathbf{X}_{j_{2}}-\mathbf{X}_{j_{1}}\right) \hat{\mathbf{e}}_{\varsigma} \mathcal{C}_{\nu, \zeta}^{(\varsigma)} \mathcal{P}_{\mu \mu ; \nu \zeta}^{\left(j_{2} ; j_{1}\right)}+i \xi \sum_{j_{3} \notin\left\{j_{1}, j_{2}\right\}} \mathcal{C}_{\mu, \eta}^{(\sigma)} \hat{\mathbf{e}}_{\sigma}^{*} \cdot G^{\prime}\left(\mathbf{X}_{j_{2}}-\mathbf{X}_{j_{3}}\right) \hat{\mathbf{e}}_{\varsigma} \mathcal{C}_{\epsilon, \zeta}^{(\varsigma)} \mathcal{P}_{\nu \nu, \mu \mu ; \epsilon \zeta}^{\left(j_{1}, j_{2} ; j_{3}\right)},
\end{aligned}
$$

where we have used the many-atom extension of Eq. (C2)

$$
\mathcal{P}_{\nu_{1} \nu_{1}, \ldots, \nu_{\ell-1} \nu_{\ell-1} ; \nu_{\ell} \zeta}^{\left(j_{1}, \ldots, j_{\ell-1} ; j_{\ell}\right)}=f_{\nu_{1}}^{\left(j_{1}\right)} \ldots f_{\nu_{\ell}}^{\left(j_{\ell}\right)} \mathcal{P}_{\nu_{\ell} \zeta}^{\left(j_{\ell}\right)}
$$

Equation (C3) represents the case (i) of Eq. (42) and we can directly construct it from Eq. (C1) by assuming the structure $(\mathrm{C} 4)$ for the correlation functions. In Eq. (C3) the two-atom internal level correlation function $\mathcal{P}_{\nu \nu ; \mu \eta}^{\left(j_{1} ; j_{2}\right)}$ is coupled to the three-atom internal level correlation function $\mathcal{P}_{\nu \nu, \mu \mu ; \epsilon \zeta}^{\left(j_{1}, j_{2} ; j_{3}\right)}$. Similarly, we can show that the three-atom internal level correlation function is coupled to the four-atom internal level correlation function. The process eventually yields a hierarchy of equations for the correlation functions.

Consider only the case (i) of Eq. (42) $(\nu=\tau$ and $\mu=\beta$ ), that is given by Eq. (C3), and the corresponding hierarchy of equations with only the analogous diagonal terms $\mathcal{P}_{\nu_{1} \nu_{1}, \ldots, \nu_{\ell-1} \nu_{\ell-1} ; \nu_{\ell} \zeta}^{\left(j_{1}, \ldots, j_{\ell-1} ; j_{\ell}\right)}$ included. In this case we can similarly extend our argument to show that Eqs. (C4) and $(\mathrm{C} 1)$ provide the full solution to the entire hierarchy of equations for internal level correlation functions. Therefore, the case (i) and all the correlation functions $\mathcal{P}_{\nu_{1} \nu_{1}, \ldots, \nu_{\ell-1} \nu_{\ell-1} ; \nu_{\ell} \zeta}^{\left(j_{1}, \ldots, j_{\ell-1} j_{\ell}\right)}$ can be solved by the classical electrodynamics simulations of Eq. (C1) and the ansatz (C4). Note that, although the correlation function for the internal levels [Eq. C4] factorizes, the atomic system still becomes correlated by light due to induced spatial correlations. The case (ii) of Eq. (42) that does not necessarily have $\nu=\tau$ and $\mu=\beta$ is notably more complicated. The light-induced coupling in that case represents virtual quantum processes that are beyond the classical electrodynamics simulation method.
[1] J. Javanainen and J. Ruostekoski, Opt. Express 24, 993 (2016), URL http://www.opticsexpress.org/ abstract. cfm?URI=oe-24-2-993.

[2] R. H. Lehmberg, Phys. Rev. A 2, 883 (1970), URL http: //link.aps.org/doi/10.1103/PhysRevA.2.883.

[3] R. H. Lehmberg, Phys. Rev. A 2, 889 (1970), URL http: //link.aps.org/doi/10.1103/PhysRevA.2.889.

[4] A. Ishimaru, Wave Propagation and Scattering in Random Media: Multiple Scattering, Turbulence, Rough Surfaces, and Remote-Sensing, vol. 2 (Academic Press, St. Louis, Missouri, 1978)

[5] B. A. van Tiggelen, A. Lagendijk, and A. Tip, J. Phys. Cond. Mat. 2, 7653 (1990).

[6] O. Morice, Y. Castin, and J. Dalibard, Phys. Rev. A 51, 3896 (1995).

[7] J. Ruostekoski and J. Javanainen, Phys. Rev. A 55, 513
(1997).

[8] J. Ruostekoski and J. Javanainen, Phys. Rev. A 56, 2056 (1997).

[9] M. Rusek, A. Orłowski, and J. Mostowski, Phys. Rev. E 53, 4122 (1996), URL http://link.aps.org/doi/10. 1103/PhysRevE.53.4122.

[10] J. Javanainen, J. Ruostekoski, B. Vestergaard, and M. R. Francis, Phys. Rev. A 59, 649 (1999).

[11] J. Ruostekoski and J. Javanainen, Phys. Rev. Lett. 82, 4741 (1999).

[12] B. Berhane and T. A. B. Kennedy, Phys. Rev. A 62, 033611 (2000), URL http://link.aps.org/doi/10. 1103/PhysRevA.62.033611.

[13] J. P. Clemens, L. Horvath, B. C. Sanders, and H. J. Carmichael, Phys. Rev. A 68, 023809 (2003), URL http: //link.aps.org/doi/10.1103/PhysRevA.68.023809. 
[14] F. A. Pinheiro, M. Rusek, A. Orlowski, and B. A. van Tiggelen, Phys. Rev. E 69, 026605 (2004).

[15] L. Chomaz, L. Corman, T. Yefsah, R. Desbuquois, and J. Dalibard, New Journal of Physics 14, 005001 (2012).

[16] S. D. Jenkins and J. Ruostekoski, Phys. Rev. B 86, 085116 (2012).

[17] S. D. Jenkins and J. Ruostekoski, Phys. Rev. A 86, 031602(R) (2012).

[18] B. Olmos, D. Yu, Y. Singh, F. Schreck, K. Bongs, and I. Lesanovsky, Phys. Rev. Lett. 110, 143602 (2013), URL http://link.aps.org/doi/10.1103/PhysRevLett.110. 143602.

[19] M. Antezza and Y. Castin, Phys. Rev. A 88, 033844 (2013), URL http://link.aps.org/doi/10. 1103/PhysRevA.88.033844.

[20] Y. Li, J. Evers, W. Feng, and S.-Y. Zhu, Phys. Rev. A 87, 053837 (2013), URL http://link.aps.org/doi/10. 1103/PhysRevA.87.053837.

[21] J. Javanainen, J. Ruostekoski, Y. Li, and S.-M. Yoo, Phys. Rev. Lett. 112, 113603 (2014).

[22] J. Pellegrino, R. Bourgain, S. Jennewein, Y. R. P. Sortais, A. Browaeys, S. D. Jenkins, and J. Ruostekoski, Phys. Rev. Lett. 113, 133602 (2014), URL http://link.aps . org/doi/10.1103/PhysRevLett.113.133602.

[23] S. E. Skipetrov and I. M. Sokolov, Phys. Rev. Lett. 112, 023905 (2014), URL http://link.aps.org/doi/ 10.1103/PhysRevLett.112.023905.

[24] R. J. Bettles, S. A. Gardiner, and C. S. Adams, Phys. Rev. A 92, 063822 (2015), URL http://link.aps.org/ doi/10.1103/PhysRevA.92.063822.

[25] S. Krämer, L. Ostermann, and H. Ritsch, arXiv:1506.09079 (2015).

[26] J. Ruostekoski, Phys. Rev. A 60, R1775 (1999), URL http://link.aps.org/doi/10.1103/PhysRevA.60. R1775.

[27] S. D. Jenkins and J. Ruostekoski, Phys. Rev. B 86, 085116 (2012).

[28] M. Segev, Y. Silberberg, and D. N. Christodoulides, Nat. Phot. 7, 197 (2013)

[29] J. D. Jackson, Classical Electrodynamics (Wiley, New York, 1999), 3rd ed.

[30] M. Born and E. Wolf, Principles of Optics (Cambridge University Press, Cambridge, UK, 1999), 7th ed.

[31] S. Balik, A. L. Win, M. D. Havey, I. M. Sokolov, and D. V. Kupriyanov, Phys. Rev. A 87, 053817 (2013).

[32] K. Kemp, S. J. Roof, M. D. Havey, I. M. Sokolov, and D. V. Kupriyanov, http://arxiv.org/abs/1410.2497 (2014).

[33] J. Keaveney, A. Sargsyan, U. Krohn, I. G. Hughes, D. Sarkisyan, and C. S. Adams, Phys. Rev. Lett. 108, 173601 (2012).

[34] T. Bienaimé, S. Bux, E. Lucioni, P. W. Courteille, N. Piovella, and R. Kaiser, Phys. Rev. Lett. 104, 183602 (2010), URL http://link.aps.org/doi/10. 1103/PhysRevLett.104.183602.

[35] J. Chabé, M.-T. Rouabah, L. Bellando, T. Bienaimé, N. Piovella, R. Bachelard, and R. Kaiser, Phys. Rev. A 89, 043833 (2014), URL http://link.aps.org/doi/10. 1103/PhysRevA. 89.043833.

[36] C. C. Kwong, T. Yang, M. S. Pramod, K. Pandey, D. Delande, R. Pierrat, and D. Wilkowski, Phys. Rev. Lett. 113, 223601 (2014), URL http://link.aps.org/doi/ 10.1103/PhysRevLett.113.223601.

[37] D. Tong, S. M. Farooqi, J. Stanojevic, S. Krishnan, Y. P.
Zhang, R. Côté, E. E. Eyler, and P. L. Gould, Phys. Rev. Lett. 93, 063001 (2004).

[38] R. Heidemann, U. Raitzsch, V. Bendkowsky, B. Butscher, R. Löw, L. Santos, and T. Pfau, Phys. Rev. Lett. 99, 163601 (2007).

[39] J. D. Pritchard, D. Maxwell, A. Gauguet, K. J. Weatherill, M. P. A. Jones, and C. S. Adams, Phys. Rev. Lett. 105, 193603 (2010).

[40] T. Wilk, A. Gaëtan, C. Evellin, J. Wolters, Y. Miroshnychenko, P. Grangier, , and A. Browaeys, Phys. Rev. Lett. 104, 010502 (2010).

[41] H. Schempp, G. Günter, C. S. Hofmann, C. Giese, S. D. Saliba, B. D. DePaola, T. Amthor, M. Weidemüller, S. Sevinçli, and T. Pohl, Phys. Rev. Lett. 104, 173602 (2010).

[42] T. Chanelière, D. N. Matsukevich, S. D. Jenkins, T. A. B. Kennedy, M. S. Chapman, and A. Kuzmich, Phys. Rev. Lett. 96, 093604 (2006).

[43] F. Lemoult, G. Lerosey, J. de Rosny, and M. Fink, Phys. Rev. Lett. 104, 203901 (2010).

[44] V. A. Fedotov, N. Papasimakis, E. Plum, A. Bitzer, M. Walther, P. Kuo, D. P. Tsai, and N. I. Zheludev, Phys. Rev. Lett. 104, 223901 (2010).

[45] S. D. Jenkins and J. Ruostekoski, New Journal of Physics 14, 103003 (2012).

[46] S. D. Jenkins and J. Ruostekoski, Phys. Rev. Lett. 111, $147401(2013)$

[47] G. Adamo, J. Y. Ou, J. K. So, S. D. Jenkins, F. De Angelis, K. F. MacDonald, E. Di Fabrizio, J. Ruostekoski, and N. I. Zheludev, Phy. Rev. Lett. 109, 217401 (2012).

[48] R. Pierrat and R. Carminati, Phys. Rev. A 81, 063802 (2010).

[49] S. Jennewein, M. Besbes, N. J. Schilder, S. D. Jenkins, C. Sauvan, J. Ruostekoski, J.-J. Greffet, Y. R. P. Sortais, and A. Browaeys, arXiv:1510.08041 (2015).

[50] S. D. Jenkins, J. Ruostekoski, J. Javanainen, R. Bourgain, S. Jennewein, Y. R. P. Sortais, and A. Browaeys, arXiv:1602.01037 (2016).

[51] J. Ruostekoski, Phys. Rev. A 61, 033605 (2000), URL http://link.aps.org/doi/10.1103/PhysRevA.61. 033605.

[52] E. A. Power and S. Zienau, Philos. Trans. R. Soc. 251, 427 (1959).

[53] R. G. Woolley, Proc. R. Soc. Lond. A 321, 557 (1971), ISSN 1364-5021, 1471-2946, URL http://rspa. royalsocietypublishing.org/content/321/1547/557.

[54] E. A. Power, Introductory Quantum Electrodynamics, vol. 24 of Mat. Phys. Ser. (Longmans, London, 1964).

[55] C. Cohen-Tannaudji, J. Dupont-Roc, and G. Grynberg, Photons and Atoms: Introduction to Quantum Electrodynamics (John Wiley \& Sons, New York, 1989).

[56] B. Bransden and C. Joachain, Physics of Atoms and Molecules (Pearson Education, Harlow, 2003).

[57] D. F. Walls and G. J. Milburn, Quantum Optics (Springer-Verlag, Berlin, 1994).

[58] E. Akkermans, A. Gero, and R. Kaiser, Phys. Rev. Lett. 101, 103602 (2008).

[59] R. J. Glauber, Phys. Rev. 130, 2529 (1963), URL http: //link.aps.org/doi/10.1103/PhysRev.130.2529.

[60] R. J. Glauber, Phys. Rev. 131, 2766 (1963), URL http: //link.aps.org/doi/10.1103/PhysRev.131.2766.

[61] P. L. Kelley and W. H. Kleiner, Phys. Rev. 136, A316 (1964), URL http://link.aps.org/doi/10.1103/ PhysRev.136.A316. 
[62] B. R. Mollow, Phys. Rev. 188, 1969 (1969), URL http: //link.aps.org/doi/10.1103/PhysRev.188.1969.

[63] T. S. Kao, S. D. Jenkins, J. Ruostekoski, and N. I. Zheludev, Phys. Rev. Lett. 106, 085501 (2011).

[64] W. S. Bakr, J. I. Gillen, A. Peng, S. Fölling, and M. Greiner, Nature 462, 74 (2009).

[65] J. F. Sherson, C. Weitenberg, M. Endres, M. Cheneau, I. Bloch, and S. Kuhr, Nature 467, 68 (2010).

[66] C. Weitenberg, M. Endres, J. F. Sherson, M. Cheneau, P. Schauß, T. Fukuhara, I. Bloch, and S. Kuhr, Nature 471, 319 (2011).
[67] O. Morsch and M. Oberthaler, Rev. Mod. Phys. 78, 179 (2006).

[68] We use here the less common notation that $\rho_{\text {ge }}$ corresponds to the matrix element $\langle e|\hat{\rho}| g\rangle$ of the density operator $\hat{\rho}$. The order of the subscripts is chosen to mirror the second-quantized formalism used in the remainder of the paper, such that $\rho_{g e}$ is related to $\left\langle\hat{\psi}_{g}^{\dagger} \hat{\psi}_{e}\right\rangle$.

[69] Note, however, that we refer to the approximation here as semiclassical, since we still include nonlinear effects due to saturation in a two-level system. 